\title{
Multimodal Physiological Signals for Workload Prediction in Robot-assisted Surgery
}

\author{
TIAN ZHOU, JACKIE S. CHA, GLEBYS GONZALEZ, and JUAN P. WACHS, \\ Purdue University \\ CHANDRU P. SUNDARAM, Indiana University School of Medicine \\ DENNY YU, Purdue University
}

\begin{abstract}
Monitoring surgeon workload during robot-assisted surgery can guide allocation of task demands, adapt system interfaces, and assess the robotic system's usability. Current practices for measuring cognitive load primarily rely on questionnaires that are subjective and disrupt surgical workflow. To address this limitation, a computational framework is demonstrated to predict user workload during telerobotic surgery. This framework leverages wireless sensors to monitor surgeons' cognitive load and predict their cognitive states. Continuous data across multiple physiological modalities (e.g., heart rate variability, electrodermal, and electroencephalogram activity) were simultaneously recorded for twelve surgeons performing surgical skills tasks on the validated da Vinci Skills Simulator. These surgical tasks varied in difficulty levels, e.g., requiring varying visual processing demand and degree of fine motor control. Collected multimodal physiological signals were fused using independent component analysis, and the predicted results were compared to the ground-truth workload level. Results compared performance of different classifiers, sensor fusion schemes, and physiological modality (i.e., prediction with single vs. multiple modalities). It was found that our multisensor approach outperformed individual signals and can correctly predict cognitive workload levels $83.2 \%$ of the time during basic and complex surgical skills tasks.
\end{abstract}

CCS Concepts: • Human-centered computing $\rightarrow$ Human computer interaction (HCI); Interactive systems and tools; User interface management systems;

Additional Key Words and Phrases: Workload prediction, robot-assisted surgery, multimodal sensing, machine learning, sensor fusion and resilience

\section{ACM Reference format:}

Tian Zhou, Jackie S. Cha, Glebys Gonzalez, Juan P. Wachs, Chandru P. Sundaram, and Denny Yu. 2020. Multimodal Physiological Signals for Workload Prediction in Robot-assisted Surgery. ACM Trans. Hum.-Robot Interact. 9, 2, Article 12 (January 2020), 26 pages.

https://doi.org/10.1145/3368589

\section{INTRODUCTION}

Current Robotic-assisted Surgery (RAS) programs at hospitals train surgeons to perform minimally invasive surgery with the latest robotic techniques and telerobotic equipment, such as the da Vinci Surgical system. This system can augment surgeons' sensorimotor capabilities and allow

Authors' addresses: T. Zhou, J. S. Cha, G. Gonzalez, J. P. Wachs, and D. Yu, Purdue University, 315 N. Grant St. West Lafayette, IN 47907, USA; emails: \{zhou338, soyoun, gonza337, jpwachs, dennyyu\}@purdue.edu; C. Sundaram, Indiana University School of Medicine, 535 N. Barnhill Dr. Indianapolis, IN 46202, USA; email: sundaram@iupui.edu.

Permission to make digital or hard copies of all or part of this work for personal or classroom use is granted without fee provided that copies are not made or distributed for profit or commercial advantage and that copies bear this notice and the full citation on the first page. Copyrights for components of this work owned by others than the author(s) must be honored. Abstracting with credit is permitted. To copy otherwise, or republish, to post on servers or to redistribute to lists, requires prior specific permission and/or a fee. Request permissions from permissions@acm.org.

(C) 2020 Copyright held by the owner/author(s). Publication rights licensed to ACM.

2573-9522/2020/01-ART12

https://doi.org/10.1145/3368589 
surgeons to perform more dexterous manipulations. However, such systems cannot yet work in an autonomous mode. Current RAS interfaces are unable to initiate or autonomously assist during operations. This limits the robot's role to being a slave with the surgeon in the role of the master. As the collaborative-robots era emerges, it is increasingly important to design robots that can take a more active role during a surgical procedure. For example, a robot's awareness of the surgeon's intraoperative workload level can facilitate the robot to provide different levels of assistance that can reduce the cognitive load of the surgeon [1] and allow for better human-robot collaboration. Some potential of assistive strategies that will be enabled with cognitive load sensing include using virtual fixtures to prevent risky motions [2], removing unintentional hand tremors [3], taking over simple and repetitive subtasks [4], and providing augmented reality cues about future steps [5]. Providing intraoperative workload feedback would also accelerate surgeon training programs $[6,7]$ by customizing training according to individual stress profiles and focusing on the specific task demands that are associated with high cognitive load [8].

To provide user-driven autonomous assistance, we first need to reliably estimate surgeons' workload levels intraoperatively. Current approaches for measuring workload predominantly rely on post-task surveys [9-14]. Although surveys are cost-effective and can be easily implemented in hospitals, the usefulness of surveys in RAS is limited, since high workload states will only be identified post-surgery after the surgeons take the survey. Some studies have tried implementing surveys intraoperatively (surgeons take the survey in the middle of the procedure). However, this can disrupt surgical workflow. The subjectivity of surveys is another limitation, since responses can vary between and within individuals. A sensor-based computational framework can overcome these limitations, and this article aims to assess intraoperative workload using wireless physiological sensors. Previous studies have correlated physiological signals to workload such as heart rate variability (HRV) [15-18], electrodermal activity (EDA) [19-21], electroencephalogram (EEG) activity [22, 23], electromyography (EMG) activity [24], and wrist motion (MOV) [25]. We propose a framework that can leverage these relationships and multiple sensing modalities to predict user workload using machine learning algorithms. The effectiveness of the framework is evaluated on a RAS dataset with twelve surgeons performing simulated tasks on the da Vinci Skills Simulator (dVSS) (Intuitive Surgical Inc., Sunnyvale, California).

This article is organized as follows. Section 2 discusses related work, and Section 3 presents the methodologies used for the workload prediction framework. Section 4 details the experiments and results, followed by discussions in Section 5. Finally, Section 6 summarizes the contributions of this work.

\section{RELATED WORK}

Four approaches have been primarily used for measuring cognitive workload and performance: questionnaires (Section 2.1), expert assessment \& annotation (Section 2.2), secondary-task performance (Section 2.3), and physiological sensors (Section 2.4). The advantages and disadvantages of each approach are summarized in Table 1. Each approach is evaluated using four different criteria relevant to RAS. Intraoperative indicates whether the approach can provide an immediate workload assessment during the surgical procedure. Objective indicates whether the approach provides objective or subjective metrics. Non-interruptive indicates whether the approach interrupts surgical workflow. Low-cost indicates resource required to implement the approach.

\subsection{Questionnaire-based Workload Assessment}

Questionnaires have been used for assessing cognitive workload in the operation room (OR) since the 1990s [35]. Usually, surgeons will be given a survey at the end of a task to rate the mental demands, physical demands, and situational stress perceived during the task. Some of the 
Table 1. Advantages and Disadvantages of the Four General Approaches for Cognitive Workload Assessment

\begin{tabular}{|c|c|c|c|c|}
\hline Approach & Intraoperative & Objective & Non-interruptive & Low-cost \\
\hline Questionnaire [10, 26]-[29] & $\times$ & $\times$ & $\times$ & $\checkmark$ \\
\hline Expert annotation [14,30] & $\checkmark$ & $\times$ & $\checkmark$ & $\times$ \\
\hline Secondary-task [12,13] & $\checkmark$ & $\checkmark$ & $\times$ & $\checkmark$ \\
\hline Sensor-based [19, 28, 31-34] & $\checkmark$ & $\checkmark$ & $\checkmark$ & $\checkmark$ \\
\hline
\end{tabular}

most widely used questionnaires include NASA Task Load Index (NASA-TLX) [9], SURG-TLX [27], Subjective Workload Assessment Technique (SWAT) [10], and Workload Profile (WP) [29]. Some studies have implemented these surveys intraoperatively, but these surveys interrupt operative flow. These disruptions are harmful and can potentially lengthen operation time and increase risks for adverse events [36-38]. Moreover, the subjective nature of the questionnaire leads to intra- and inter-subject variability, making it an inconsistent metric [14]. In summary, questionnaire-based assessment techniques are disruptive, subjective, and only available post-task, thus making them inadequate for the scenario discussed in this article.

\subsection{Expert Annotations for Workload Assessment}

Many assessment tools require subject matter experts for evaluating surgical performance (mainly for technical skills), such as the Objective Structured Assessment of Technical Skill (OSATS) [30] and Global Operative Assessment of Laparoscopic Skills [39]. To apply these tools, experts either observe the surgery in the OR or use recorded videos to estimate of surgeons' performance. Recent studies have used this expert-observer approach to intervene and improve surgical outcomes via surgical coaches. However, the need for experts presents logistical challenges (e.g., expert availability) and is resource intensive (high costs and time) [14]. Early attempts have been made to use crowdsourcing techniques to reduce the cost and difficulty of surgery skill evaluation with OSATS, but this is still at preliminary stages and limited to post-operative assessment [40]. Finally, although this approach has demonstrated success in evaluating surgeon performance, another limitation to the observer-based approach is the difficulty in reliably estimating cognitive load through observations. These factors limit the potential impact and implementation of this approach.

\subsection{Secondary Tasks for Workload Assessment}

Secondary tasks assess the amount of "spare" mental capacity by asking users to perform two tasks simultaneously. The primary task is the task of interest (e.g., surgical skill, technique, or subtask of the procedure), and a secondary task is performed alongside the primary task. For example, a visual secondary task (i.e., ball-and-tunnel) was used to evaluate mental workload in simulated laparoscopic surgery [12]. Specifically, the surgeon manipulated the laparoscope's tooltip (primary task) while being queried on the ball movements superimposed over the primary task (secondary task). Although this technique showed significant differences in workload between Fundamentals of Laparoscopic Surgery tasks and cadavers tasks [6], this approach is extremely intrusive since the secondary task requires reallocation of mental resources (e.g., attention). In surgery, this may impacts patient safety, thus not feasible with live patients. In Reference [13], an interval production task was proposed as a secondary task to measure surgeons' mental workload. The interval production task required the subject to make a response each time when they believe a target time interval has elapsed (e.g., between 3 and 30 seconds). The accuracy and consistency of the reported time interval can be used to estimate mental workload, and such technique was believed to be less intrusive than traditional secondary tasks, since the subject voluntarily uttered 
the response. Again, the major drawback for secondary task is distraction to the surgeons and potential decrements on surgery performance and patient outcome.

\subsection{Sensor-based Workload Assessment}

The introduction of wireless, miniaturized, and low-powered sensors for human physiological sensing provides an approach to quantify surgeons' cognitive states more objectively and with less interruption than the assessments above. Specifically, several physiological signals have been associated with workload, e.g., EEG, EDA, and HRV.

EEG has been correlated with workload in many domains outside of healthcare [41, 42]. In surgery [43], EEG signals were used to objectively differentiate between novice and expert surgeons and revealed significant differences in cognitive metrics between the two groups. EEG signals were also used to identify cognition patterns of a surgical mentor and trainee when teaching RAS skills. It was found that the mentor's workload increased when the trainee's workload decreased [8].

HRV and EDA have also been correlated to changes in cognitive load. HRV captures the variation of time intervals between heartbeats and is negatively correlated with workload in several domains (e.g., human-computer interaction [47, 48], aviation [49, 50] and navigation [42, 51]). EDA (also known as Skin Conductance Response) measures sweat gland activity that is regulated by the sympathetic nervous systems. EDA has been used as an index of mental stress and workload $[44,45]$. For example, the total number of EDA peaks and EDA amplitude increases with higher workload [21, 46].

Previous work suggests that physiological signals are promising for stress and workload assessment [52]. The comparisons between major sensor-based approaches for workload assessment is summarized in Table 2. The modalities column indicates the sensors used for workload recognition. Fusion refers to the technique used for sensor fusion, if applicable. The analytic models describe the method used to recognize workload (e.g., correlation, regression, or classification models). Baseline indicates how ground-truth values for the workload was determined. Although the raw groundtruth workload values have a continuous range, many studies discretized the raw scores into two [31], three [53, 55], or four levels $[34,56]$ for training the classification algorithms to predict workload. Subjects describes the sample size and background of the participants. And finally, area (task) refers to the scenario and the task studied in each paper.

\subsection{Summary}

This article addresses the following key gaps in our ability to objectively evaluate workload:

Multimodality: While multimodal fusion and predictive analysis for workload have been used in domains such as HCI and driving, current techniques for measuring workload in complex teleoperation tasks during RAS have been limited to a single modality and basic statistical/correlation analyses. This article investigates multimodal sensing and sensor fusion, together with predictive modeling in the area of RAS.

Generalization: Previous studies in RAS either study workload assessment with a single surgeon and multiple tasks [33] or with a single task and multiple surgeons [28]. This article focuses on the generalization capability of the workload prediction across multiple surgeons (12) and multiple tasks (6).

Resilience: New physiological sensors are wireless, and there are data quality risks from interference. The robustness of our algorithm was evaluated at varying levels of noise.

Contributions: This article makes the following contributions: (1) a multimodal workload prediction algorithm in the RAS domain; (2) exploration of the Dempster-Shafer Theory of uncertainty 
Table 2. Comparison with State-of-the-art Sensor-based Approaches for Workload Assessment ${ }^{1}$

\begin{tabular}{|c|c|c|c|c|c|c|c|}
\hline Paper & Year & Modalities & Fusion & $\begin{array}{c}\text { Analytic } \\
\text { models }\end{array}$ & Baseline & Subjects & $\begin{array}{c}\text { Area } \\
(\text { task) }\end{array}$ \\
\hline Wilson [54] & 2003 & EEG, ECG, EOG & feature concat. & neural network & $\begin{array}{c}\text { post-task } \\
\text { survey }\end{array}$ & $\begin{array}{c}7 \\
\text { subjects }\end{array}$ & $\begin{array}{c}\text { HCI } \\
\text { (MATB) }\end{array}$ \\
\hline $\begin{array}{l}\text { Solovey } \\
\text { [31] }\end{array}$ & 2014 & $\begin{array}{c}\text { HRV, EDA, car } \\
\text { speed and steering } \\
\text { angle }\end{array}$ & feature concat. & $\begin{array}{l}\text { five common } \\
\text { classifiers }\end{array}$ & $\begin{array}{l}\text { level of } \\
\text { n-back }\end{array}$ & $\begin{array}{c}120 \\
\text { drivers }\end{array}$ & $\begin{array}{c}\text { HCI } \\
\text { (driving) }\end{array}$ \\
\hline Novak [32] & 2014 & $\begin{array}{l}\text { EEG, HRV, EDA, } \\
\text { eye tracking, arm } \\
\text { movement, task } \\
\text { score }\end{array}$ & feature concat. & $\begin{array}{l}\text { linear regression, } \\
\text { random forest }\end{array}$ & NASA-TLX & $\begin{array}{c}10 \\
\text { students }\end{array}$ & $\begin{array}{c}\text { HRI } \\
\text { (games) }\end{array}$ \\
\hline Guru [33] & 2015 & EEG & not applicable & $\begin{array}{c}\text { correlation } \\
\text { analysis }\end{array}$ & NASA-TLX & $\begin{array}{c}1 \\
\text { surgeon }\end{array}$ & RAS cases \\
\hline $\mathrm{Yu}[28]$ & 2017 & IMU & not applicable & t-tests, ANOVA & SURG-TLX & $\begin{array}{c}10 \\
\text { surgeons } \\
\end{array}$ & RAS cases \\
\hline Zhang [53] & 2017 & EEG and ECG & $\begin{array}{l}\text { interactive } \\
\text { mutual } \\
\text { information } \\
\text { modeling }\end{array}$ & classification & $\begin{array}{l}\text { level of } \\
n \text {-back }\end{array}$ & $\begin{array}{c}10 \\
\text { students }\end{array}$ & $\begin{array}{c}\text { HCI } \\
\text { (n-back) }\end{array}$ \\
\hline $\begin{array}{c}\text { Aghajani } \\
{[34]}\end{array}$ & 2017 & EEG, fNIRS & PCA concat. & $\begin{array}{c}\text { support vector } \\
\text { machine }\end{array}$ & $\begin{array}{c}\text { task } \\
\text { difficulty }\end{array}$ & $\begin{array}{c}17 \\
\text { students } \\
\end{array}$ & $\begin{array}{c}\mathrm{HCI} \\
\text { (n-back) }\end{array}$ \\
\hline Liu [55] & 2017 & $\begin{array}{c}\text { EEG, fNIRS, HRV, } \\
\text { breath rate and } \\
\text { depth }\end{array}$ & $\begin{array}{l}\text { Naïve-Bayes } \\
\text { fusion }\end{array}$ & $\begin{array}{c}\text { multiclass linear } \\
\text { discriminant } \\
\text { analysis }\end{array}$ & $\begin{array}{l}\text { level of } \\
n \text {-back }\end{array}$ & $\begin{array}{c}21 \\
\text { subjects }\end{array}$ & $\begin{array}{c}\text { HCI } \\
\text { (n-back) }\end{array}$ \\
\hline $\begin{array}{c}\text { Jimenez- } \\
\text { Molina } \\
\text { [56] }\end{array}$ & 2018 & $\begin{array}{l}\text { EDA, ECG, PPG, } \\
\text { EEG, temperature, } \\
\text { and pupil dilation }\end{array}$ & feature concat. & $\begin{array}{l}\text { multinomial } \\
\text { logistic } \\
\text { regression, } \\
\text { multi-class } \\
\text { support vector } \\
\text { machine, and } \\
\text { multi-layer } \\
\text { perceptron }\end{array}$ & $\begin{array}{l}\text { Pupil } \\
\text { dilation }\end{array}$ & $\begin{array}{c}61 \\
\text { students }\end{array}$ & $\begin{array}{l}\text { HCI (web } \\
\text { browsing) }\end{array}$ \\
\hline Ours & 2018 & $\begin{array}{c}\text { EEG, EDA, HRV, } \\
\text { MOV, EMG }\end{array}$ & $\begin{array}{c}\text { early/late } \\
\text { fusion, DST }\end{array}$ & $\begin{array}{l}\text { six modern } \\
\text { classifiers }\end{array}$ & $\begin{array}{l}\text { NASA-TLX } \\
\text { and task } \\
\text { difficulty }\end{array}$ & $\begin{array}{c}12 \\
\text { residents }\end{array}$ & RAS cases \\
\hline
\end{tabular}

${ }^{1}$ Explanation of Acronyms: Electroencephalogram (EEG), Electrocardiogram (ECG), Electrooculography (EOG), Heart Rate Variability (HRV), Electrodermal Activity (EDA), Inertial Measurement Unit (IMU), functional Near-Infrared Spectroscopy (fNIRS), Photoplethysmogram (PPG), Electromyography (EMG), Movement (MOV), Principal Component Analysis (PCA), Dempster-Shafer Theory (DST), Analysis of Variance (ANOVA), NASA Task Load Index (NASA-TLX), Human-Computer Interaction (HCI), Multi-Attribute Task Battery (MATB), Human-Robot Interaction (HRI), Robot-Assisted Surgery (RAS).

for sensor fusion and modeling; (3) investigation of the relationship between objective workload ground-truth (task difficulty) and subjective workload ground-truth (NASA-TLX); (4) evaluation of the algorithm's generalization across multiple surgeons and tasks; and (5) examination of the algorithm's resilience to noise.

\section{METHODS}

This section presents the different techniques used in the proposed computational framework for cognitive workload assessment. First, it gives an overview of the system and the simulation scenarios, then it discusses multimodal feature extraction, ground-truth determination, feature inspection and ranking, workload prediction algorithms, and finally Dempster-Shafer Theory for sensor fusion and modeling. 


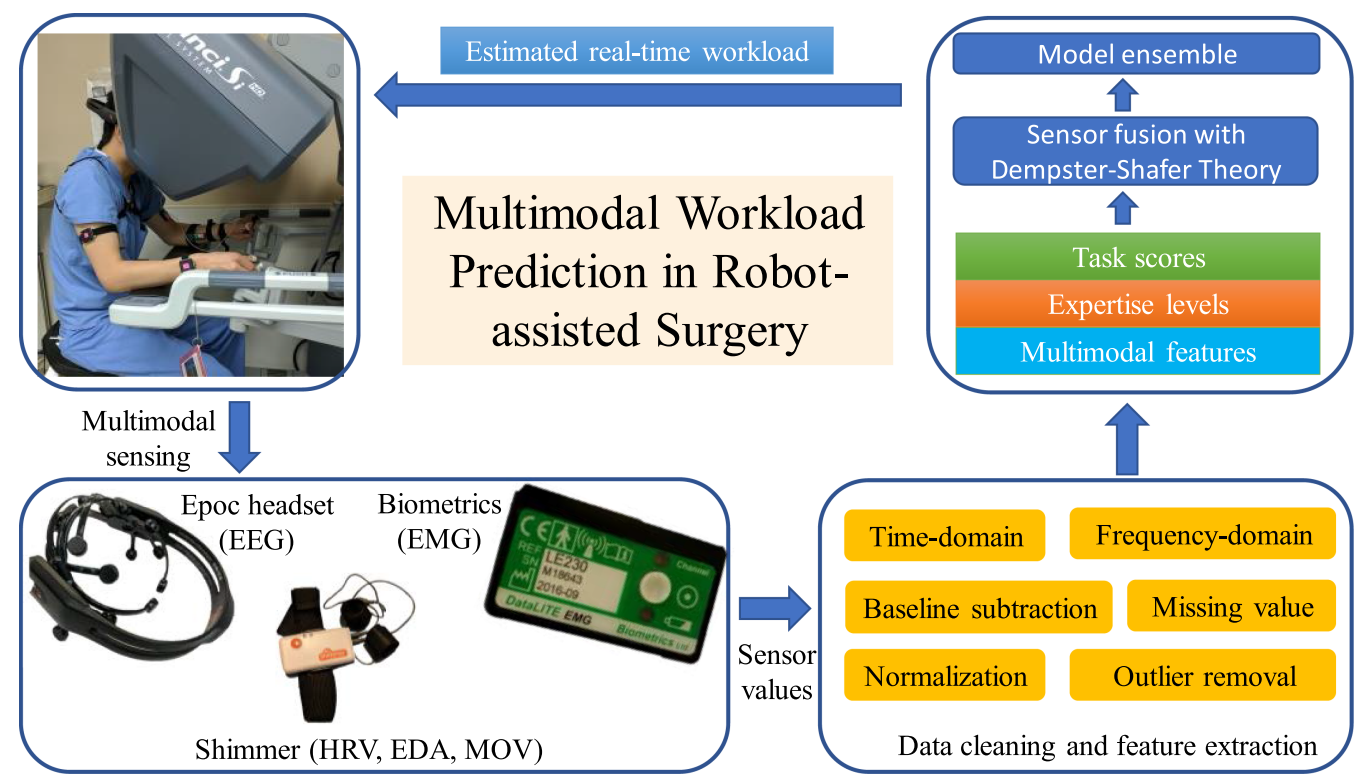

Fig. 1. Interaction system overview, showing the da Vinci master console, the surgeon, the various sensors, and the workload prediction pipeline.

\subsection{System Overview}

In RAS, the surgeon sits in front of the robotic console and interacts with navigation controllers with both hands for a prolonged period of time (e.g., RAS prostatectomy procedures have been observed to average two hours [28]). The end-effectors on the robotic arms mirror the motions of the surgeon's hands and perform operations directly inside the patient. In our scenario, the dVSS was used, where the surgeon interacted with the master console (console was identical to the one used in live procedures) to perform surgical training tasks in a simulation setting. The surgeon was monitored through a set of sensors that captured his/her physiological signals in real-time as they performed the virtual surgical task at the console. The sensor measurements were analyzed by signal processing modules and sent to the workload reasoning engine for inference of current workload level. An overview of the entire system with the hardware and software components is illustrated in Figure 1.

\subsection{Study Protocol}

This work was overseen by the Institutional Review Board of all academic and medical institutions involved. A total of 12 experienced subjects were recruited to participate in this study. The subjects were urology residents $(N=12)$, with ages ranging from 26 to 38 (mean 30, std. dev. is 3 , and $67 \%$ were males). This study was part of the resident training program curriculum; thus, these simulation sessions were directly tied to their training, and participants were especially motived to succeed. The residents varied in their post-medical school experiences, which ranged from one to eight years. Multiple wireless sensors were worn by the participant to measure EEG, EMG, HRV, EDA, and MOV signals. Sensors were calibrated according to manufacturer instructions. Participants then completed the simulated surgical tasks on the dVSS. The tasks were pre-programmed in the dVSS system by the robotic surgery manufacturer (Intuitive Surgical, Inc.). Six surgical training tasks were selected through consultation from a surgeon with extensive surgical education experience (Figure 2). The selected tasks covered a wide range of difficulty levels to simulate varying 

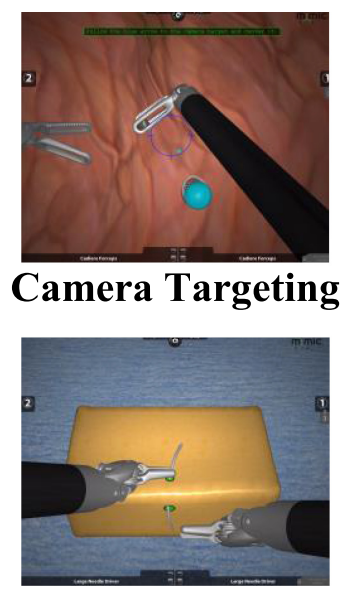

Suture Sponge

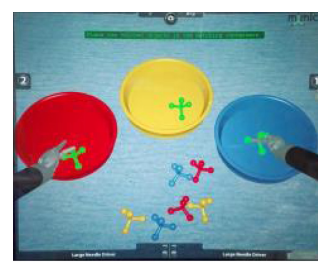

Pick and Place

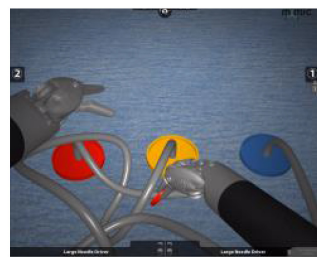

Ring and Rail

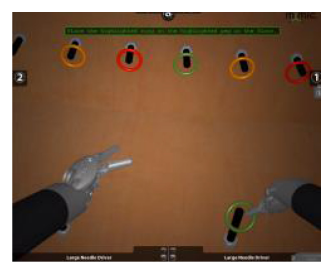

Peg Board

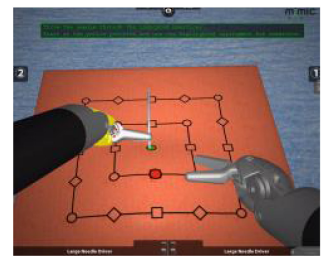

Dots and Needles

Fig. 2. Visualization of the six surgical tasks selected from the dVSS powered by Mimic Simulation [57].

levels of workload demands. Each participant performed all six tasks with up to three different difficulty levels (further described in later sections). In the remainder of the article, we denote the completion of one surgical task with any difficulty level as a "session" (indexed by $k$ ). Each participant performed multiple sessions with different task types and different difficulty levels. The specific number of sessions each participant performed is relative to their own progress in the training curriculum, and thus, not the same for each participant. Overall, participants performed an average of 9.83 sessions per person with a standard deviation of 1.86. After finishing each session, participants completed the gold-standard NASA-TLX survey to report their self-perceived workload levels during the session. Participants also completed a demographics survey. From the 12 participants, a total of $119(K=119)$ surgical task sessions were acquired and formed the base dataset for analysis. Each task session formed the time window to analyze workload variations. We assumed that the average workload level was consistent within session and reflected the overall workload demand of the entire session. This assumption was reasonable as each task focused on one skills metric (e.g., camera control or clutching) and did not vary within the session. Also, the participants' responses on the NASA-TLX were a summary of the workload for the entire task, so the constant workload assumption was necessary to align the session time-scale with the gold-standard measure (NASA-TLX). Thus, we believed the assumption of sustained workload was appropriate (limitation will be discussed in Section 5.5).

\subsection{Multimodality and Feature Crafting}

3.3.1 Epoc Headset for EEG Signals. The Emotiv Epoc headset (๔Emotiv, San Francisco, CA) was used to measure EEG. The wireless headset was worn on the head to capture EEG signals at $128 \mathrm{~Hz}$. The sensor captured 14 channels at the following locations: AF3, F7, F3, FC5, T7, P7, O1, O2, P8, T8, FC6, F4, F8, and AF4. The F, P, and O bands were found relevant to workload levels in previous work [32, 54, 58]. EEG signals were typically noisy and susceptible to artifacts due to blinks, head movements, and facial expressions. To reduce these effects, we limited the slew rate to $-15 \mu V$ to $15 \mu V$ to remove occasional noise spikes [59], applied an Infinite Impulse Response (IIR) high-pass filter with feedforward filter coefficients of $0.008 \mathrm{~Hz}$ and feedback filter coefficients of $0.992 \mathrm{~Hz}$ [60], and used a Hanning window filter of 2 seconds to remove fast Fourier transform (FFT) wrap-around step artifacts [61]. After the raw signals were cleaned, they were converted 
into the frequency domain using FFT. According to the literature in cognitive sciences, different frequency bands were associated with different cognitive processes of the brain [62]. We followed the pipelines proposed in Reference [63] to convert the power spectrum into five frequency bands: theta $(4-8 \mathrm{~Hz})$, alpha $(8-12 \mathrm{~Hz})$, low-beta $(12-16 \mathrm{~Hz})$, high-beta $(16-25 \mathrm{~Hz})$, and gamma bands $(25-$ $40 \mathrm{~Hz}$ ). We then extracted statistical features, including the mean, standard deviation, minimum, maximum, and median values from the bin power of each band as features, following References $[64,65]$. Such process resulted in 14 (channels) ${ }^{*} 4$ (bands) ${ }^{*} 5$ (descriptors) $=280$ (EEG features) for session $k$, denoted as $\tilde{X}_{k}^{E E G} \in \mathbb{R}^{280}$.

3.3.2 Shimmer for EDA, MOV and HRV Signals. The Shimmer3 GSR+ Unit (๑Shimmer, Dublin, Ireland) measured the EDA with two electrodes placed on the index and middle fingers at a sampling rate of $204.8 \mathrm{~Hz}$. Continuous decomposition analysis was performed on Ledalab [66, 67] with $0.01 \mu \mathrm{S}$ minimum amplitude. Three features were used to characterize EDA following [68], which were the mean, standard deviation of amplitude, and the number of peaks. Such process resulted in 3 EDA features for session $k$, denoted $\tilde{X}_{k}^{E D A} \in \mathbb{R}^{3}$.

Wrist motion signals were also obtained using accelerometers and gyroscope on the Shimmer3. The mean and standard deviation of three-axis gyroscope, three-axis low-noise accelerometer $( \pm 2 \mathrm{~g})$, and three-axis wide-range accelerometer $( \pm 16 \mathrm{~g})$ were obtained for feature representation [69], totaling $\{\text { mean, std }\}^{*}\{$ three-axis $\}$ \{gyroscope, low-noise accelerometer, wide-range accelerometer $\}=18$ features. For task session $k$, the MOV features were denoted as $\tilde{X}_{k}^{M O V} \in \mathbb{R}^{18}$.

Heart rate variability was estimated using optical pulse ear-clip on the Shimmer3. Kubios HRV Standard software (Kubios Oy॰ , Kuopio, Finland) was used to extract time-domain and frequencydomain features. In the time-domain, the standard deviation of NN interval (SDNN) and root mean square of successive difference (RMSSD) were extracted following [70]. For frequency-domain, the power of very-low frequency (VLF, $0-0.04 \mathrm{~Hz}$ ), low frequency (LF, 0.04-0.15Hz), high frequency (HF, $0.15-0.4 \mathrm{~Hz}$ ), total frequency (TF, $0-0.4 \mathrm{~Hz}$ ), and ratio of LF power over HF power were used [71]. This process resulted in a total of $7 \mathrm{HRV}$ features for session $k$, denoted as $\tilde{X}_{k}^{H R V} \in \mathbb{R}^{7}$.

3.3.3 Biometrics for EMG Signals. DataLITE wireless surface EMG sensors (Biometrics $\odot$, Ltd, Newport, UK) were used to measure upper-body muscle activity at a sampling rate of $2 \mathrm{kHz}$. Eight sensors were placed along the neck to forearm: on the left and right splenius capitis, trapezius, biceps brachii, and brachioradialis. The amplitude and the power mean and median were calculated features for each EMG channel. For task session $k$, the EMG features were denoted as $\tilde{X}_{k}^{E M G} \in \mathbb{R}^{32}$.

3.3.4 Curating the Dataset. As described previously, the analysis was conducted over the entire task execution/session procedure, e.g., from the beginning to the end of a surgical training task. This was necessary as the ground-truth workload levels (based on subjective questionnaires) can only be established for a complete session, rather than just segments, and these ground-truth levels were necessary as a comparison baseline. Even though the experiments in this article were conducted offline after the operations were finished, the resulting predictive model can be deployed to assess surgeon workload in real-time and intraoperatively. However, translation to real-time will be addressed in the future work section.

For session $k$, the combined features are denoted as $\tilde{X}_{k}=\left[\tilde{X}_{k}^{E E G}, \tilde{X}_{k}^{E D A}, \tilde{X}_{k}^{M O V}, \tilde{X}_{k}^{H R V}, \tilde{X}_{k}^{E M G}\right] \in$ $\mathbb{R}^{M}$ where $M=340$ was the sum of all feature dimensions. All the $M$ features of $\tilde{X}_{k}$ were normalized into the range of $[0,1]$ for further comparisons. The normalized signal was denoted as $X_{k}$ and was used in the final analysis.

\subsection{Workload Prediction}

Given the feature descriptors $X_{k} \in \mathbb{R}^{M}$ obtained from the physiological signals, this analysis aimed to estimate $\hat{Y}_{k}=\phi\left(X_{k}\right) \in\{0,1\}$ to link physiological signals with the surgeon workload. 
3.4.1 Independent Component Analysis (ICA). The candidate features were highly redundant and exhibit collinearity [72], posing challenges for analysis. To address this problem, ICA was used to separate the independent components from highly redundant and correlated measurements. It has been commonly used for EEG signal processing to separate out artifacts embedded in the data [73]. Given a multi-dimensional input $X_{k} \in \mathbb{R}^{M}$, ICA mapped features into $m$ independent components $Z_{k} \in \mathbb{R}^{m}$, which were mutually independent from each other. These can be used to reconstruct the original signal $X_{k}$. Based on different sensor fusion schemes, ICA can either be applied to the concatenated raw signal $X_{k}$ to get independent components $Z_{k}=I C A_{m}\left(X_{k}\right) \in \mathbb{R}^{m}$ or to signals from each individual modality $X_{k}^{E E G}, X_{k}^{E M G}, X_{k}^{M O V}, X_{k}^{E D A}$, and $X_{k}^{H R V}$ to get separate components $Z_{k}^{E E G}, Z_{k}^{E M G}, Z_{k}^{M O V}, Z_{k}^{E D A}$, and $Z_{k}^{H R V}$.

3.4.2 Sensor Fusion. Sensor fusion was the process of integrating information from multiple sensors. Fusion can be performed at different stages of the processing pipeline, resulting in different strategies. In this article, we explored early fusion and late fusion, together with different dimension reduction and feature selection techniques. The different fusion schemes are shown in Figure 3. For figure clarity, we illustrated the fusion scheme with only three modalities, but all five modalities were used in the actual algorithm.

Early Fusion: This process concatenated the raw features together (i.e., $X_{k}=$ $\left[X_{k}^{E E G}, X_{k}^{E M G}, X_{k}^{M O V}, X_{k}^{E D A}\right.$, and $\left.\left.X_{k}^{H R V}\right] \in \mathbb{R}^{M}\right)$ and then built a predictor for the fused signal, i.e., $\hat{y}_{k}=\phi\left(X_{k}\right)$. Early fusion can preserve potential interactions between the raw features and result in a richer representation of the original features [74].

Early Fusion with ICA first performed the ICA analysis on the concatenated features. It retained the advantage of early fusion and removed redundancy in the feature representation. This fusion scheme was formulated as

$$
\hat{y}_{k}=\phi\left(Z_{k}\right)=\phi\left(I C A_{m}\left(X_{k}\right)\right) .
$$

In the following, we used the subscript to indicate the number of independent components in ICA (e.g., ICA $\mathrm{I}_{5}$ indicates the usage of five independent components).

Early Fusion with Feature Selection (FS) performed feature selection on the concatenated features before classification. In our scenario, we used the significance values ( $p$-value) from the two-sampled t-test for feature selection. More specifically, the p-value of each feature with the ground-truth (high-low workload) was calculated first. A cutoff p-value (0.05) was used to select predictive features. We also enforced a minimum number of retained feature (3) and a maximum number of retained feature (treated as hyper-parameter and tuned with cross-validation). In the rest of the article, we used the format "FS (minimum features, maximum features, cutoff p-value)" to represent different FS configurations, e.g., FS (3, 30, 0.05).

Late Fusion referred to the scheme where individual predictions were made from each modality separately, and then those were combined via a voting scheme. This process would be described by the formula

$$
\hat{y}_{k}=\text { vote }\left\{\hat{y}_{k}^{E E G}, \hat{y}_{k}^{E M G}, \hat{y}_{k}^{M O V}, \hat{y}_{k}^{E D A}, \hat{y}_{k}^{H R V}\right\}
$$

where $\hat{y}_{k}^{E E G}=\phi\left(X_{k}^{E E G}\right)$ and similarly for EMG, MOV, EDA, and HRV. Late fusion can be more robust to errors caused by hardware malfunction or lack of precision than early fusion, since each predictor can work independently, thus the algorithm is more resilient. In this article, we explored two voting schemes, the average voting and the Dempster-Shafer Theory (DST) voting [75]. The average voting referred to the process where the probability outputs from different classifiers were averaged to make the final decision. In the DST fusion scheme, the output probability for each classifier was used to initialize a basic belief mass, and those masses generated from different classifiers 


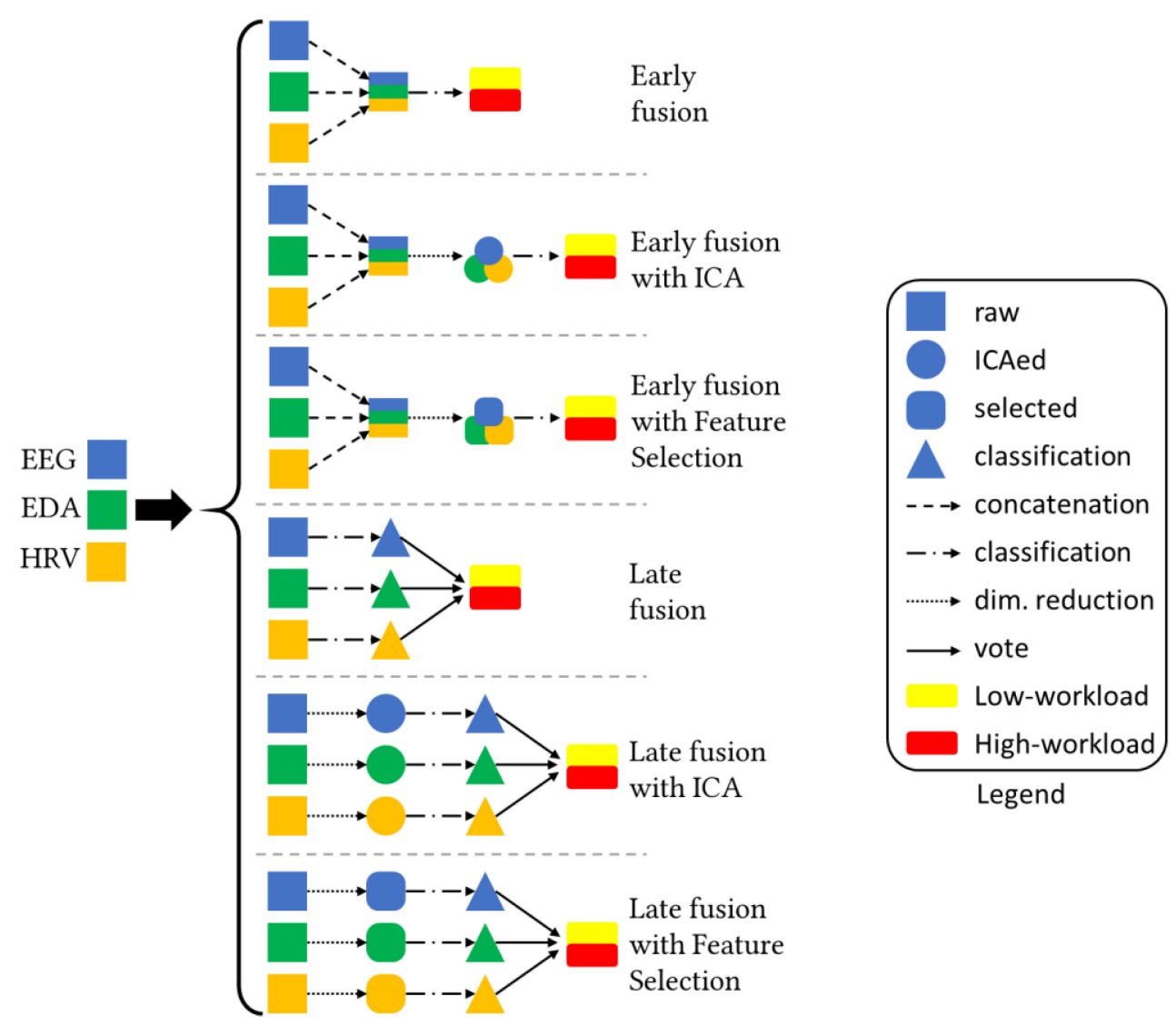

Fig. 3. The different fusion schemes discussed in this article. Different colors indicate different modalities (here, we only showed three instead of five for figure simplicity). Different shapes represent signals of different stages in the processing pipeline, where squares represent the original raw features $\left(X_{k}\right)$, circles represent the signals after ICA analysis $\left(Z_{k}\right)$, and triangles represent the predictor result $\left(\hat{\boldsymbol{y}}_{k}\right)$. For the meaning of arrows and decisions, refer to the figure legend.

were combined using Dempster Rule of Combination (DRC) [76]. The DRC rule considered the agreement and conflict between different beliefs to make a final decision, which was denoted $\hat{y}_{k}$.

Late Fusion with ICA performed ICA on raw features of each modality, obtaining independent decisions from each modality and then combining them via a voting scheme. This process can be described by the formula

$$
\hat{y}_{k}=\operatorname{vote}\left\{\hat{y}_{k}^{E E G}, \hat{y}_{k}^{E M G}, \hat{y}_{k}^{M O V}, \hat{y}_{k}^{E D A}, \hat{y}_{k}^{H R V}\right\},
$$

where $\hat{y}_{k}^{E E G}=\phi\left(Z_{k}^{E E G}\right)=\phi\left(I C A_{m}\left(X_{k}^{E E G}\right)\right)$ and similarly for EMG, MOV, EDA, and HRV.

Late Fusion with Feature Selection (FS) performed feature selection individually on features from each modality, trained separate classifiers, and then used a voting scheme in the end to get a final decision.

3.4.3 Supervised Learning for Prediction. The last step in the prediction pipeline was to instantiate the predictor $\phi(\cdot)$ with a supervised machine learning algorithm. To accomplish this, 
we compared the performance of six classifiers: Support Vector Machines (SVM), Decision Trees (DT), Random Forest (RF), Extra Trees (ET), Gradient Boosting Trees (GBT), and Adaboost (AB).

\section{EXPERIMENTS AND RESULTS}

This section presents the experimental setup and results as follows: (1) determine the cognitive workload ground-truth, (2) correlate the multimodal features with the ground-truth, and (3) present results for different machine learning experiments, including base classifier selection, modality importance, sensor fusion selection, and robustness to noise.

\subsection{Cognitive Workload Ground-truth}

Framework development required ground-truth knowledge to train the workload prediction algorithm. Ground-truth labels were determined using perceived workload (i.e., NASA-TLX) and task difficulty. Both were used, since workload can be influenced by both a participant's perception and the underlying demands required of the task. Specifically, subjective perception was commonly used to measure perceived workload as ground truth (e.g., NASA-TLX [32, 33], SURG-TLX [28], or general surveys [54]). Other studies used the underlying task difficulty to determine the workload level (e.g., using level of n-back [31, 34] and intrinsic task parameters [77]). Both approaches had strengths and weaknesses. Demands required to complete task goals directly affect the amount of workload imposed on the subject. However, due to individual differences in experience/expertise/perception, the same task can result in significantly different workload levels for different subjects. These individual variations can be captured by surveys. Therefore, this article leveraged the advantages of both the subjective and objective ground-truth as detailed in the following sections. The ground-truth was discretized as binary, i.e., either high workload or low workload. Although binarization reduced the granularity of the measure, this simplification was highly relevant and desired for clinical applications. Specifically, the overall clinical goal was to prevent cognitive overload that can lead to human error; thus, accurately detecting "high" workload can help inform the design and implementation of future real-time interventions.

4.1.1 Subject-perceived Workload (NASA-TLX). The perceived workload was collected via the NASA-TLX survey completed by the participant at the end of each surgical task session (i.e., after finishing any of the surgical tasks shown in Figure 2). Perceived workload levels were assessed using the six subscales of the NASA-TLX: mental demand, physical demand, temporal demand, performance, effort, and frustration. Each scale was rated from 0 (very low) to 10 (very high). The subscales were then averaged to create an overall score [78]. Although the original NASA-TLX then apply weights to each subscale, many studies validated the unweighted approach for the NASATLX [78]. The resultant metric represented the overall workload level and was named Overall Task Load Index (OTLX), denoted as $O_{k} \in[0,1]$ (for session $k$ ). The histogram of the OTLX scores from all the participants $(K=119)$ is shown in Figure 4, together with the fitted Gamma distribution to show skewness. As shown, the histogram had a peak around 0.25 and was right-skewed. The OTLX had a grand mean of 0.425 , a median of 0.375 , and a standard deviation of 0.202 .

Since the OTLX score was used to calculate the workload ground-truth, it was important to conduct a closer examination the metric over different conditions, i.e., Subjects, Orders, Tasks, Expertise, and Score. This analysis can reveal the underlying interaction between the OTLX score and the various factors to better generate a consensus workload ground-truth.

The five conditions under investigation were Subjects, Orders, Tasks, Expertise, and Score. The subjects ID ranged from 1 to 12 , since we had 12 participants. The Orders was the order in which the task session was completed by the subject. The maximum value for Orders was 12, as that was the maximum amount of sessions completed by a subject. The Tasks was an ID for surgical task 


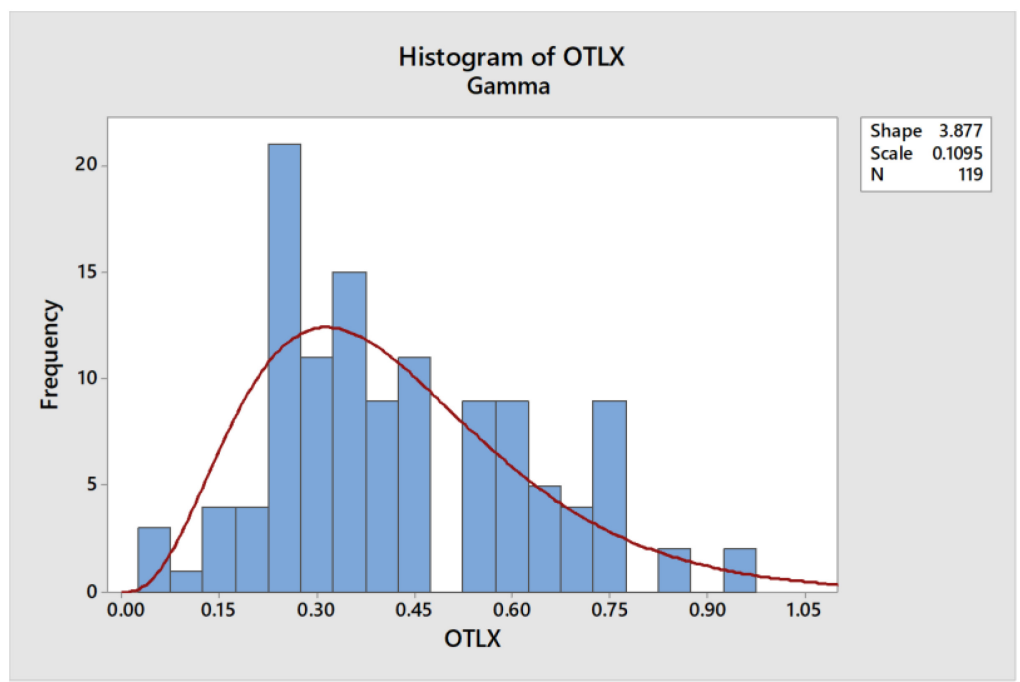

Fig. 4. Histogram of the OTLX from all participants $(K=119)$, the fitted Gamma distribution is displayed.

Table 3. Summary of the Test Results for OTLX Over Different Conditions

\begin{tabular}{|c|c|c|c|}
\hline$\downarrow$ Condition\Tests $\rightarrow$ & Levene's equal variance test & ANOVA's test of equal means & Post hoc test \\
\hline Subjects & $\mathrm{F}(11,107)=2.52, \mathrm{p}=0.007$ & $\mathrm{~F}(11,40.3)=35.15, \mathrm{p}<0.001$ & Five groups (Games-Howell) \\
\hline Orders & $\mathrm{F}(11,107)=0.66, \mathrm{p}=0.774$ & $\mathrm{~F}(11,107)=2.38, \mathrm{p}<0.05$ & One group (Tukey) \\
\hline Tasks & $\mathrm{F}(5,113)=1.59, \mathrm{p}=0.169$ & $\mathrm{~F}(5,113)=5.33, \mathrm{p}<0.001$ & Three groups (Tukey) \\
\hline Expertise & $\mathrm{F}(5,113)=7.05, \mathrm{p}<0.001$ & $\mathrm{~F}(5,48.04)=21.79, \mathrm{p}<0.001$ & Three groups (Games-Howell) \\
\hline Scores & \multicolumn{3}{|c|}{ Pearson's correlation coefficient $-0.386(\mathrm{p}<0.001)$} \\
\hline
\end{tabular}

completed, ranging from 1 to 6 where 1 represented Camera Targeting and 6 represented Dots and Needles (Figure 2). The Expertise represented the post-grad years, and we had six values (1, 2, 3, $4,6,8)$ with min 1 and max 8. The Score represented surgeon performance the finished session. This score was a continuous value from 0 to 100 and was calculated by dVSS based on the task completion time, number of instrument collisions, economy of motion, and excessive end-effector forces, and so on [79].

The procedure for analyzing the effect of each condition (Subjects, Orders, Tasks, and Expertise) on OTLX was the same for all five conditions. First, Levene's test of equal variance was conducted on the OTLX score using each condition as a factor. If the equal-variance assumption was satisfied (large p-value in the Levene's test), then regular ANOVA tests were conducted followed by Tukey's post hoc tests. If the equal-variance assumption was violated (small p-value in the Levene's test), then the Welch's ANOVA was then performed followed by Games-Powell post hoc test, as these do not require equal variance assumption. The grouping result of the post hoc test was represented with grouping letters $(\mathrm{A} \sim \mathrm{E})$, where groups that do not share any overlapping letters had significantly different means. For example, group A was significantly different from group $B C$, while group A was not different from group $\mathrm{AB}$ (since they shared the same letter A). For the Score condition, since it was a continuous value instead of categorical, we calculated the Pearson's correlation coefficient between Score and OTLX.

The statistical results are summarized in Table 3, and the interval plots with post hoc grouping information are shown in Figure 5. The factors Subjects, Tasks, and Expertise all induced different 

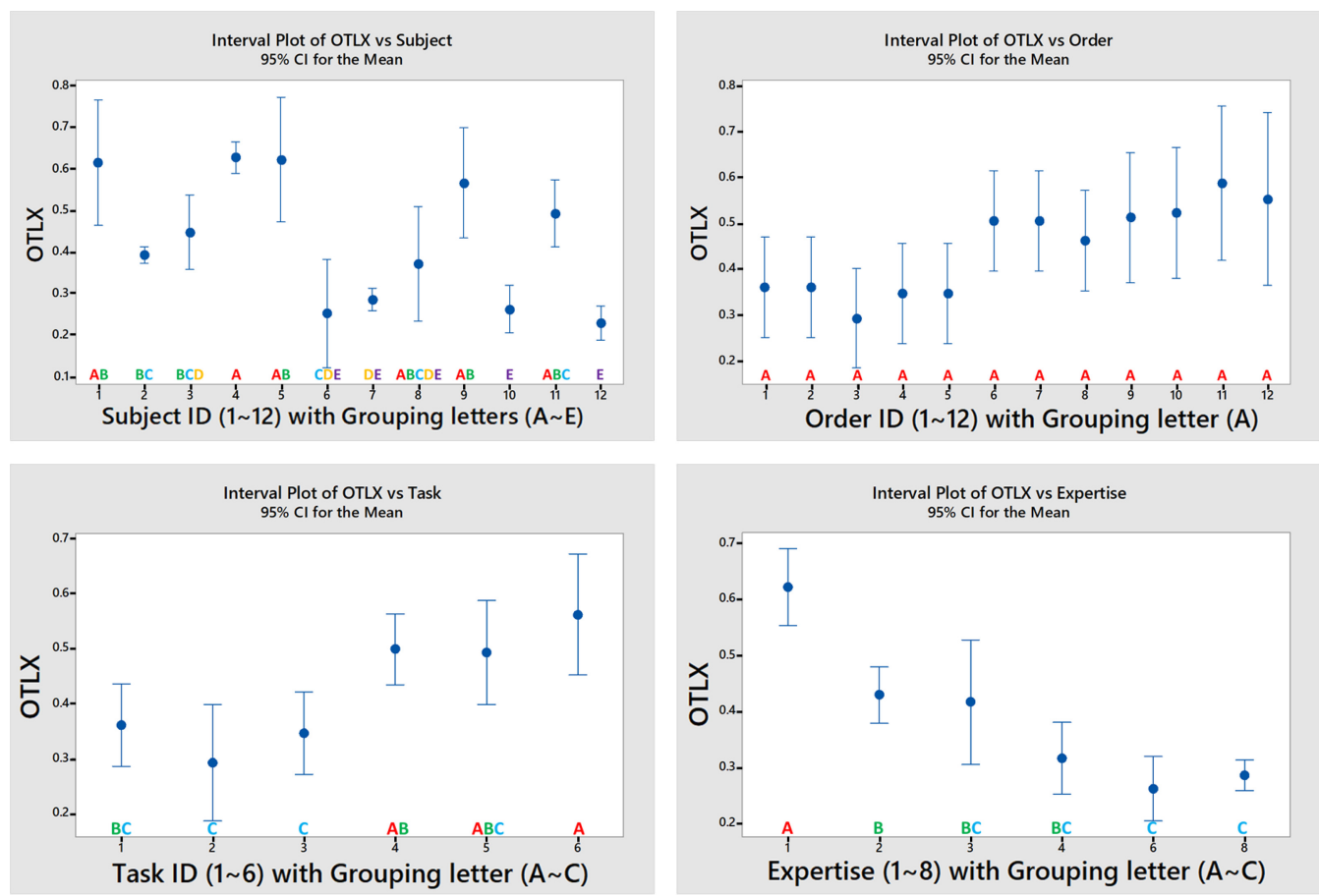

Fig. 5. Interval plot of OTLX vs. Subjects (top left), Order (top right), Tasks (bottom left), and Expertise (bottom right). The grouping information as determined by the post hoc test is shown with letters (A E) above the $\mathrm{x}$-axis legend. The groups who do not share any overlapping letters have significantly different means.

groups of OTLX levels, implying the potential effect of Subjects, Tasks, and Expertise on perceived workload levels (OTLX). However, the factor Orders only outputted one group, meaning that the subject's OTLX scores did not change over the course of the experiment. This observation may suggest that potential order effects (like fatigue) were not present in this study (this further supports that there was consistent engagement and workload for the participants). Regarding Scores, a significantly negative correlation was found, indicating that lower performance scores correlate to higher OTLX scores, a relationship shown in previous literature [80]. However, Scores information cannot be acquired intraoperatively in the current da Vinci system, thus it is a post-task metric that can only be used for off-line analysis and not a suitable factor for goal of real-time assistance.

4.1.2 Task-determined Workload. As seen on the Task interval plot (Figure 5, bottom-left), tasks were observed to induce different workload levels. Therefore, the following approach was used to determine ground-truth workload levels based on task characteristics. Different tasks evaluated different RAS skill sets, and Table 4 summarized the skills that each simulation task assessed [81]. The checkmark $(\checkmark)$ indicated the primary skill evaluation and asterisk $\left({ }^{*}\right)$ indicated secondary skill evaluations [81]. The top row in Table 4 represented the difficulty score (on a scale from 1 to 10) for each skill determined by two clinical subject matter experts. Difficulty scores for each task were combined with corresponding primary/additional skill scores. Each primary skill was assigned a weight of 1 , and each additional skill was assigned a weight of 0.5 . The weighted difficulty score was shown in the right-most column. The average of all the weighted scores was 5.67, and this cutoff threshold was used to categorize each task into a binary high/low workload level. 
Table 4. The Primary/secondary Skillsets Evaluated in each Simulation Task, and the Weighted Difficulty Score

\begin{tabular}{|c|c|c|c|c|c|c|c|}
\hline Difficulty score & 2 & 2 & 2 & 6 & 4 & 7 & \\
\hline Metrics $\rightarrow$ & Endowrist & Camera & Clutching & Needle & Needle drive & Needle drive & Weighted \\
\hline$\downarrow$ Simulation tasks & manipulation & control & & control & - basic & - advanced & difficulty score \\
\hline Camera targeting 1 & & $\checkmark$ & & & & & 2 \\
\hline Camera targeting 2 & & $\checkmark$ & * & & & & 3 \\
\hline Pick and Place & $\checkmark$ & & & & & & 2 \\
\hline Peg Board 1 & $\checkmark$ & & & & & & 2 \\
\hline Peg Board 2 & $\checkmark$ & * & * & & & & 4 \\
\hline Suture Sponge 1 & * & & & * & $\checkmark$ & & 8 \\
\hline Suture Sponge 2 & * & * & & * & $\checkmark$ & & 9 \\
\hline Suture Sponge 3 & * & * & & * & & $\checkmark$ & 12 \\
\hline Ring and Rail 1 & $\checkmark$ & * & & & & & 3 \\
\hline Ring and Rail 2 & $\checkmark$ & * & * & & & & 4 \\
\hline Dots and Needles 1 & * & & & * & $\checkmark$ & & 8 \\
\hline Dots and Needles 2 & * & & & * & & $\checkmark$ & 11 \\
\hline
\end{tabular}

$\checkmark$ represents primary skill focus; *represents additional skill focus.

This binary level was indicated by $Y_{k}^{\text {task }} \in\{0,1\}, k=1, \ldots, 119$ for each of the task sessions. As mentioned before, this ground-truth binarization step was a necessary first-step to build a classification model for predicting workload levels. With this binarization approach, Suture Sponge and Dots and Needles were in the high workload group, while the other four tasks fell into the low workload group. Each task had a consistent low/high level, regardless of the multiple difficulty levels (i.e., all difficulty levels of the same task share the same low/high level).

4.1.3 Consensus Using Objective and Subjective Ground-truth. The final workload for each session was determined by a consensus of objective and subjective workload ground-truth. For the OTLX score $O_{k} \in[0,1]$, the grand mean $(0.425)$ was used to generate a binary workload level, denoted as $Y_{k}^{\text {OTLX }}$, where $Y_{k}^{\text {OTLX }}=1$ if $O_{k}>0.425$ and $Y_{k}^{\text {OTLX }}=0$ otherwise. To account for subject effects in OTLX scores (as shown in Table 3 and Figure 5), we conducted a per-subject normalization process similar to Reference [82]-which also removed subjective variations by normalizing the score to the each subject's average-to get Normalized Overall Task Load Index (NOTLX), denoted as $\mathrm{NO}_{k}$. This way, a robust and consistent ground-truth cognitive level was determined. The $N O_{k}$ was calculated as follows. First, each session $k$ performed by subject $n(n=1, \ldots, N)$ was denoted as $n=\sigma(k) \in\{1, \ldots, N\}$. Then, the average OTLX score for each subject was calculated, denoted as $\mu_{n}(n=1, \ldots, N)$ :

$$
\mu_{n}=\frac{\sum_{k=1,}^{K} \sigma(k)=n}{O_{k}}, n=1, \ldots, N .
$$

Then, the NOTLX score $\mathrm{NO}_{k}$ was calculated as

$$
N O_{k}=\frac{O_{k}}{\mu_{n}} \in[0,1], n=\sigma(k), k=1, \ldots, K .
$$

Finally, the $N O_{k}$ was compared to the subject-average $\mu_{\sigma(k)}$ to generate a binarized workload level $Y_{k}^{N O T L X}$ where $Y_{k}^{N O T L X}=1$ if $N O_{k}>\mu_{\sigma(k)}$ and $Y_{k}^{\text {NOTLX }}=0$ otherwise.

This resulted in three sets of ground-truth baselines, $Y_{k}^{\text {task }}, Y_{k}^{\text {OTLX }}$, and $Y_{k}^{N O T L X}$ (all binary $\in\{0,1\}$ ), each from a different perspective. To assess the agreement among these three sets, we conducted 
Table 5. Pairwise Comparison Between the Three Sets of Ground-truth $\left(Y_{k}^{\text {task }}, Y_{k}^{O T L X}, Y_{k}^{N O T L X}\right)$

\begin{tabular}{|c|c|c|}
\hline Pairs & $\chi^{2}$ test of independence & Cohen's kappa test \\
\hline$Y_{k}^{\text {task }}$ vs $Y_{k}^{\text {OTLX }}$ & $p=0.03$ & $\kappa=0.214$ \\
\hline$Y_{k}^{\text {task }}$ vs $Y_{k}^{\text {NOTLX }}$ & $p<0.001$ & $\kappa=0.590$ \\
\hline$Y_{k}^{\text {OTLX }}$ vs $Y_{k}^{\text {NOTLX }}$ & $p<0.001$ & $\kappa=0.339$ \\
\hline
\end{tabular}

Table 6. The Top-ranked Features (Based on p-values)

\begin{tabular}{|c|c|c|c|c|c|c|}
\hline Modality & Rank & Features & $\boldsymbol{\mu} \pm \boldsymbol{\sigma}$ (low workload) & $\boldsymbol{\mu} \pm \boldsymbol{\sigma}$ (high workload) & Relation & $\mathbf{p}$ \\
\hline \multirow{4}{*}{ EEG } & 1 & O2_StdDev_theta & $436.87 \pm 142.70$ & $281.85 \pm 90.46$ & negative & $<0.0001$ \\
\cline { 2 - 7 } & 2 & O1_Min_lowBeta & $202.86 \pm 40.91$ & $155.58 \pm 37.09$ & negative & $<0.0001$ \\
\cline { 2 - 7 } & 3 & P7_Max_theta & $252.73 \pm 53.63$ & $194.87 \pm 46.90$ & negative & $<0.0001$ \\
\hline \multirow{4}{*}{ EDA } & 1 & PeakCount & $9.75 \pm 14.11$ & $16.98 \pm 17.96$ & positive & 0.0320 \\
\cline { 2 - 7 } & 2 & Amplitude_Mean & $0.15 \pm 0.25$ & $0.24 \pm 0.31$ & positive & 0.1330 \\
\cline { 2 - 7 } & 3 & Amplitude_SD & $0.05 \pm 0.08$ & $0.07 \pm 0.10$ & positive & 0.2812 \\
\hline \multirow{4}{*}{ HRV } & 1 & Freq_LF/HF Power & $3.47 \pm 0.16$ & $3.53 \pm 0.13$ & positive & 0.0479 \\
\cline { 2 - 7 } & 2 & Time_RMSSD & $5.96 \pm 3.85$ & $6.76 \pm 4.00$ & positive & 0.2831 \\
\cline { 2 - 7 } & 3 & Time_SDNN & $8.42 \pm 6.04$ & $9.41 \pm 5.54$ & positive & 0.3653 \\
\hline \multirow{4}{*}{ MOV } & 1 & Accel_LN_Z_SD & $1.98 \pm 0.73$ & $3.13 \pm 1.08$ & positive & $<0.0001$ \\
\cline { 2 - 7 } & 2 & Accel_WR_Z_SD & $2.04 \pm 0.75$ & $3.13 \pm 1.02$ & positive & $<0.0001$ \\
\cline { 2 - 7 } & 3 & Accel_LN_Z_Mean & $4.72 \pm 1.50$ & $3.29 \pm 1.68$ & negative & 0.0002 \\
\hline \multirow{4}{*}{ EMG } & 1 & RN_Pwr_Mean & $67.83 \pm 26.01$ & $84.91 \pm 30.07$ & positive & 0.0016 \\
\cline { 2 - 7 } & 2 & RN_Pwr_Med & $55.17 \pm 15.84$ & $63.73 \pm 18.79$ & positive & 0.0100 \\
\cline { 2 - 7 } & 3 & RN_Amp_Med & $59.60 \pm 10.46$ & $63.82 \pm 8.13$ & positive & 0.0153 \\
\hline
\end{tabular}

Grey color indicates non-significance with cutoff p-value 0.05 .

the $\chi^{2}$ test of independence and the Cohen's Kappa test among each annotation pair (Table 5). There was a moderate agreement between $Y_{k}^{\text {task }}$ vs. $Y_{k}^{N O T L X}$, and a fair agreement between the other pairs ( $Y_{k}^{\text {task }}$ vs. $Y_{k}^{O T L X}$ and $Y_{k}^{O T L X}$ vs. $\left.Y_{k}^{N O T L X}\right)$ [83]. These results showed the diversity and consensus among the three ground-truth baselines. A majority vote was performed among the three ground-truth labels to reach a consensus, following: $Y_{k}=1$ if $Y_{k}^{\text {task }}+Y_{k}^{\text {OTLX }}+Y_{k}^{\text {NOTLX }} \geq 2$ and $Y_{k}=0$ otherwise. Following this rule, there were a total of 53 high workload sessions $\left(Y_{k}=1\right)$ and 66 low workload sessions $\left(Y_{k}=0\right)$. The percentage of high workload sessions $\left(Y_{k}=1\right)$ for each task was 8.3\% (Camera Targeting), 0\% (Pick and Place), 8.3\% (Peg Board), 90.9\% (Suture Sponge), 53\% (Ring and Rail), and 100\% (Dots and Needles). Only Ring and Rail task generated a diverse result for workload levels (53\%), and all the other tasks had a common agreement (either near $0 \%$ or near $100 \%$ ). This can be due to the endowrist manipulation skill focus on the Ring and Rail task. Although it was a relatively low difficulty technique, it required manual dexterity with robotic controllers; a skill that may be unfamiliar and therefore challenging for some participants.

\subsection{Statistical Tests for Candidate Features Sets}

T-tests were performed for each of the $M$ features to better find features associated with high and low workload levels. The p-values were used to rank all the candidate features, where a smaller p-value was ranked higher due to larger significance levels among the two groups [84]. The number of significant features (cutoff p-value of 0.05) were (with the total number of features after 
backslash): EEG (156/280), EDA (1/3), HRV (1/7), MOV (7/18), EMG (4/32). The EEG modality had the greatest number of significant features out of all the modalities. The top- 3 candidate features for each modality are shown in Table 6.

\subsection{Computational Experiment Setup}

The goal of this experiment was to evaluate the performance for predicting workload using the collected dataset of the $K$ sessions. Each session had a multimodal feature descriptor $\left(X_{k} \in \mathbb{R}^{M}\right)$ and a workload level $\left(Y_{k} \in\{0,1\}\right)$. The predicted workload level was denoted as $\hat{Y}_{k}=\phi\left(X_{k}\right) \in\{0,1\}$ with the ground-truth workload level $Y_{k}$. Four metrics were used to evaluate the prediction performances (accuracy, precision, recall, and F1 score) [85]. They were calculated as the following: True Positive (TP) $=\sum_{k=1}^{K} \hat{Y}_{k} Y_{k}$, False Positive (FP) $=\sum_{k=1}^{K} \hat{Y}_{k}\left(1-Y_{k}\right)$, True Negative (TN) = $\sum_{k=1}^{K}\left(1-\hat{Y}_{k}\right)\left(1-Y_{k}\right)$, False Negative $(\mathrm{FN})=\sum_{k=1}^{K}\left(1-\hat{Y}_{k}\right) Y_{k}$, Precision $(\mathrm{P})=T P /(T P+F P)$, Recall $(\mathrm{R})=T P /(T P+F N)$, and F1 score $(\mathrm{F} 1)=2 P R /(P+R)$. The Precision $(\mathrm{P})$ evaluated the percentage of true high workload sessions out of all reported high workload sessions, and the Recall (R) evaluated the percentage of true high workload sessions that have been correctly identified. The F1 score combined Precision and Recall into a single measurement. In the following analysis, the four metrics were reported; however, the F1 score was used as the single metric to compare model performances (F1 summarized both precision and recall and was robust to class imbalance). The evaluation of the algorithm was based on the leave-one-subject-out (loso) cross validation scheme. In each of the 12 folds (due to 12 subjects), feature descriptors from 11 subjects were used to train the model. The trained model was then evaluated on the held-out subject's data to get an accuracy measurement. In the end, the fold-averaged accuracies were used to summarize the performance of each individual fold. The loso evaluation scheme can evaluate the algorithm's generalization capability on unseen subjects. The multiple hyper-parameters for each classifier were tuned using grid-search approach based on the loso scheme [86], where the training data was separated into 11 splits based on subject ID. A classifier was trained on all but one split and validated on the held-out split. This process was repeated for each of the 11 splits, and the hyper-parameters generating the best average validation performances were selected. A final model was trained on the entire training split (11 subjects' data) with the optimal hyper-parameters, and then tested on the held-out testing split.

Several factors in the workload prediction framework needed to be tuned, such as the base classifier, the right mix of modality, the right feature selection method, and sensor fusion techniques. We took the series approach to test one factor at a time, and in each next step, used the best-found factors from the previous steps. This approach allowed for testing multiple factors and generating fast and efficient evaluations. In future work, experiments on all the factors together is needed to fully exploit the potential factor interactions on the pipeline.

\subsection{Base Classifier Selection}

This experiment evaluated the performance of different machine learning algorithms for workload prediction. The six classifiers (Table 7) were compared. To focus only on the effect of base predictors, the early fusion scheme was used, and ICA was disabled. Within-group cross-subject grid-search approach was used to find the optimal hyper-parameters for each classifier [32]. The performance of the different predictors is shown in Table 7. The SVM classifier generated the best performance with an accuracy of 0.773 and a F1 score of 0.716 . Therefore, it was used as the base classifier in the remaining analyses.

The optimal hyper-parameters found for SVM are furthered detailed for future reference as follows. Both the RBF kernel and liner kernel were tested. The $\mathrm{C}$ value for linear kernel and the $\mathrm{C}$ and 
Table 7. Performance of Six Different Classifiers and Chance as Base Predictor

\begin{tabular}{|c|c|c|c|c|}
\hline Classifier & Accuracy & Precision & Recall & F1 score \\
\hline Chance (flip a coin) & 0.487 & 0.435 & 0.509 & 0.470 \\
\hline Support Vector Machines (SVM) & $\mathbf{0 . 7 7 3}$ & $\mathbf{0 . 8 1 0}$ & $\mathbf{0 . 6 4 1}$ & $\mathbf{0 . 7 1 6}$ \\
\hline Decision Trees (DT) & 0.714 & 0.694 & 0.641 & 0.667 \\
\hline Random Forest (RF) & 0.739 & 0.739 & 0.641 & 0.687 \\
\hline Extra Trees (ET) & 0.756 & 0.800 & 0.603 & 0.688 \\
\hline Gradient Boosting Trees (GBT) & 0.731 & 0.784 & 0.547 & 0.644 \\
\hline Adaboost (AB) & 0.681 & 0.653 & 0.603 & 0.627 \\
\hline
\end{tabular}

Table 8. Performance of Different Modalities When Used Individually

\begin{tabular}{|c|c|c|c|c|}
\hline Input modality & Accuracy & Precision & Recall & F1 score \\
\hline Chance & 0.487 & 0.435 & 0.509 & 0.470 \\
\hline EEG & $\mathbf{0 . 7 8 2}$ & $\mathbf{0 . 7 5 5}$ & $\mathbf{0 . 7 5 5}$ & $\mathbf{0 . 7 5 5}$ \\
\hline EDA & 0.479 & 0.395 & 0.321 & 0.354 \\
\hline HRV & 0.504 & 0.442 & 0.434 & 0.438 \\
\hline MOV & 0.664 & 0.710 & 0.415 & 0.524 \\
\hline EMG & 0.580 & 0.526 & 0.566 & 0.545 \\
\hline
\end{tabular}

gamma value for RBF kernel were optimized. The initial range was selected based on recommendations from the scikit-learn library guidelines and spaced at a multiplication of 10 (so the grid was $10^{-3}, 10^{-2}, 10^{-1}$, etc.) and then extended if the optimal value was found at the boundary. As a result, the optimal hyper-parameter was RBF kernel with a $C$ value of $10^{2}$ and a gamma value of $10^{-5}$.

\subsection{Modality Importance}

This experiment evaluated the importance of different modalities. There was a total of five modalities: EEG, EMG, EDA, HRV and MOV. To control the experiment, no feature selection or ICA was used, and SVM was used as the base classifier for all modalities. Table 8 shows the performance of each modality. The EEG sensor delivered the best performance with an F1 score of 0.755 , followed by EMG $(\mathrm{F} 1=0.545)$, and MOV $(\mathrm{F} 1=0.524)$. The HRV $(\mathrm{F} 1=0.438)$ and EDA $(\mathrm{F} 1=0.354)$ performance were below chance performance.

\subsection{Sensor Fusion Scheme}

This experiment compared different fusion schemes (early, late, DST) and different feature selection methods as described earlier (t-test-based feature selection and ICA). There were several hyper-parameters to tune, including the number of independent components (i.e., $m$ ), the minimum/maximum allowed features, and cutoff $\mathrm{p}$-values. They were all tuned using cross-validation. For consistency, all five modalities and the SVM classifier were used. The final performance is shown in Table 9. As shown, the early fusion with $\mathrm{ICA}_{10}$ yielded the best performance, except the precision score, where Late (DST) $+\mathrm{ICA}_{5}$ generated the highest score. Since the F1 score was used to compare performances, the early fusion with $\mathrm{ICA}_{10}$ was select the best feature vector for the remaining analyses.

\subsection{Robustness to Noise During RAS}

The presence of noise may influence the robustness of our approach during RAS. Certain fusion schemes were more susceptible to noise; therefore, the purpose of this experiment was to evaluate 
Table 9. Performance of Different Fusion Schemes and Feature Selection Approaches

\begin{tabular}{|c|c|c|c|c|c|}
\hline Fusion & Feature Selection & Accuracy & Precision & Recall & F1 score \\
\hline Chance & Chance & 0.487 & 0.435 & 0.509 & 0.470 \\
\hline Early & $\mathrm{NA}$ & 0.773 & 0.810 & 0.641 & 0.716 \\
\hline Early & ICA $_{10}{ }^{*}$ & $\mathbf{0 . 8 3 2}$ & 0.811 & $\mathbf{0 . 8 1 1}$ & $\mathbf{0 . 8 1 1}$ \\
\hline Early & $\mathrm{FS}\left(3,30^{*}, 0.05\right)$ & 0.765 & 0.805 & 0.623 & 0.702 \\
\hline Late (average) & $\mathrm{NA}$ & 0.765 & 0.778 & 0.660 & 0.714 \\
\hline Late (DST) & $\mathrm{NA}$ & 0.782 & 0.787 & 0.698 & 0.740 \\
\hline Late (DST) & $\mathrm{ICA}_{5}{ }^{*}$ & 0.798 & $\mathbf{0 . 9 1 4}$ & 0.604 & 0.727 \\
\hline Late (DST) & FS (3, 30, 0.05$)$ & 0.706 & 0.750 & 0.509 & 0.607 \\
\hline
\end{tabular}

$\left(^{*}\right)$ This parameter was selected using cross-validation.

the performance of workload prediction when different levels of Gaussian noise were introduced in the sensor measurements. For early fusion and late fusion, all five modalities were used. The added Gaussian noise had zero mean and a standard deviation of $\sigma$. Two metrics were used to evaluate the resilience in performance: one was the absolute accuracy and the other was Performance Retainment Rate (PRR), which was defined as the percentage of the accuracy achieved with noise $(\sigma \neq 0)$ divided the accuracy without noise $(\sigma=0)$. The values of $\sigma$, the resultant absolute accuracy, and PRR values are shown in Appendix (Table A1 and A2). The right-most column shows the average of each row excluding the no-noise condition. As shown by both metrics, the late fusion with DST showed the highest robustness to noise, since it exhibited the highest average F1 score (0.738) and PRR values (99.76\%).

\section{DISCUSSIONS}

\subsection{Performance of Different Modalities}

When comparing the performance of each individual modality, EEG achieved the best F1 score (0.755). This suggests that EEG is the most predictive modality for characterizing workload levels. Other studies in domains outside of RAS [55, 87-90] also concluded that EEG was the salient modality for workload characterization. In RAS, EEG may be especially reliable due to the design of the dVSS. The console layout fixes the surgeon's head and upper torso in a mostly static position during the entire operation. This constrained motion may enable the delivery of reliable and high-quality EEG readings that are less susceptible to noise. Although it had the highest accuracy, the EEG sensor was also the most uncomfortable sensor due to the long set-up time and contact pressure from the electrodes. A statement from an experienced surgeon who participated in many data collection studies indicated that "in about $50 \%$ of the longer surgeries, the sensor resulted in a mild headache, which resolved after the sensor was removed."

An F1 score of 0.811 was achieved when incorporating all sensor modalities. This value falls within the upper range of classification accuracies reported in domains outside of RAS, which varied from $45 \%$ to $90 \%$ [55, 87-90]. These differences may be due to experiment designs, baseline selections, and task demands. Although desired classification accuracy levels for RAS remain unknown, McCrea et al. [91] proposed that an acceptable classification accuracy is $70 \% \sim 80 \%$ for affective video games. Our presented framework meets this accuracy threshold; however, we caution that meeting this threshold may still be insufficient for our intended RAS applications domain. Specifically, poor accuracy in clinical applications may result in alarm fatigue, usability issues, and potential patient harm. In simulation-based training on the dVSS where risks of patient harm are minimal, however, the current algorithm may have acceptable accuracy. 
Table 10. Counts of Different Characteristics (Channel on Left, Descriptor on Middle, Band on Right) for the Top 20 EEG Features

\begin{tabular}{|c|c|}
\hline Channel & Count \\
\hline O1 & 7 \\
\hline O2 & 4 \\
\hline P8 & 3 \\
\hline F3 & 3 \\
\hline AF4 & 2 \\
\hline P7 & 1 \\
\hline
\end{tabular}

\begin{tabular}{|c|c|}
\hline Descriptor & Count \\
\hline max & 6 \\
\hline mean & 4 \\
\hline median & 4 \\
\hline std & 4 \\
\hline min & 2 \\
\hline
\end{tabular}

\begin{tabular}{|c|c|c|}
\hline Band & Frequency & Count \\
\hline Theta & $4-8 \mathrm{~Hz}$ & 7 \\
\hline Alpha & $8-12 \mathrm{~Hz}$ & 6 \\
\hline Low-beta & $12-16 \mathrm{~Hz}$ & 6 \\
\hline High-beta & $16-25 \mathrm{~Hz}$ & 1 \\
\hline
\end{tabular}

The F1 score with sensor fusion was only 0.056 higher than the EEG-only scenario, and previous work suggested that accuracy differences below $10 \%$ would likely not be noticed by users [91]. The significance of this difference likely depends on the specific use case and application domain. For safety-critical areas such as surgeon workload prediction during life-threating surgery or pilot workload prediction during combat, this improvement may be critical. However, for use cases such as web browsing and VR/AR gaming, this difference in performance might not be necessary and the extra sensors may have undesirable impact on usability and algorithmic complexities. Overall, the specific sensor selection and combination is dependent on the final use case. Continuous improvement in accuracy and focus groups on acceptable accuracy for RAS are still needed.

There are several reasons why the other sensors perform worse than the EEG in assessing workload. One potential reason is that current sensing technologies are not capable of capturing the subtle differences reflected by workload changes as well as the EEG. Specifically, task demands in robotic surgery require information processing and mental demand; thus, brain activity may be more relevant to workload detection in RAS than heart rate or muscle activity. Other potential reasons include signal strength, signal noise, and/or frequent motions at the location of sensor placement. For example, EMG sensors may not be accurate for predicting workload as the tasks required low force and/or static muscle contractions. EDA could be potentially affected due to frequent finger movements and pressure changes while interacting with the robot controls. Although this work showed that other sensors were less effective than EEG, these modalities still warrant further exploration due to potential cost and usability benefits, e.g., HRV was easy to obtain, lowcost, and integrated in many consumer devices (e.g., armbands and watches).

\subsection{A Closer Examination of EEG Features}

EEG channel was the most promising modality to workload prediction. A closer look at the most relevant EEG features showed the impact of electrode locations, frequency bands, and statistical descriptor. Table 10 summarizes the characteristics of EEG features for the top 20 EEG features ranked by $\mathrm{p}$-values (all p-values are $<0.001$ ).

The occipital (O) channels were found to be highly predictive of workload levels. This was similar to findings reported in [32], which focused on analyzing EEG signals for workload prediction in human-robot interaction scenarios. For descriptors, the max descriptor was the most frequent. For frequency bands, the frequencies between $4-16 \mathrm{~Hz}$ (i.e., theta, alpha, low-beta) were more relevant to workload estimation, and these findings aligned with results reported in References [54, 92]. Although our results aligned with many current studies, other studies reported relationships between EEG and workload that were not observed in the current study. For example, Reference [58] suggested that the $\mathrm{POz}$ channel was the most accurate channel in predicting workload during numerical learning tasks (arithmetic calculation), and Reference [93] showed that Cz, P3, and 
Pz channels were significant locations for detecting mental workload. A potential explanation for these discrepancies is the differences in scenario tasks (numerical learning vs. tele-surgery). Although EEG responses can be task-dependent, the current study provides data for the RAS-specific tasks.

\subsection{Multimodal Fusion Schemes}

Without using ICA, multimodal fusion (both early and late) performed worse than the bestperforming individual modality (i.e., EEG). This suggests that adding more features without proper processing and dimension reduction can decrease overall performance. When adding ICA into early fusion, the performance significantly increased with an F1 score of 0.811 and $5.6 \%$ higher than the EEG-only performance. When examining the different voting schemes for late fusion, the DST-based approach with and without ICA was found to be better than simply averaging the likelihood. Such an outcome indicates that DST is a promising method for late sensor fusion.

\subsection{Task Workload and Subjective Workload}

In this article, participants were asked to perform six different surgical tasks at varying difficulty levels. As detailed in the methods, some of these tasks were more complex and demanding than others. These inherent task differences may influence the interpretation of our model and suggest that our sensing approach is measuring inter-task differences instead of cognitive workload. However, three factors can further clarify the intended contribution of the sensing approach.

First, although isolation of task and workload effects are not feasible for the RAS tasks used in the current study (these tasks were actual tasks designed for surgeon training), previous controlled studies on physiological signals and workload have shown that physiological measures are predictive of cognitive workload. Specifically, these studies demonstrate this fundamental relationship with basic psychomotor vigilance computer tasks and memory tasks in a controlled laboratory setting (see references in Section 2.4).

Second, it is widely accepted that contributors to workload are multifactorial and difficult to separate for real tasks performed in actual field environments (especially technically complex tasks seen in surgery training). Workload demands are influenced by (1) actions needed to achieve the goals of the tasks (e.g., movement distances, accuracy requirement, visual search requirements, and number of actions required) and (2) the subjectively perceived demands (e.g., fatigue and other mental states, stress, technical skill, and variation in cognitive capacity). In this article, the training pipeline used for the final workload prediction model considered both the subjective component (determined by self-reported surveys, see Section 4.1.1) and the task component (determined by experts based on task demands, see Section 4.1.2).

Finally, movement and muscle activation patterns were expected to capture inter-task differences (e.g., motion can differ when moving a camera versus placing a suture). However, the motor indicators (i.e., EMG and MOV) were actually poor predictors of workload in our results. This may be due to the high cognitive demands (e.g., EEG planning and perception) that the trainee study population is likely under, thus motor skills was not a great indicator of the cognitive demands. Although this potentially limits application of this algorithm to robotic training, future work is still needed to find a way to cultivate a larger physiological dataset and more diverse populations (e.g., from experienced surgeons where skilled movements may be a strong predictor) to better reflect these interactions between task and perceived workload.

\subsection{Robustness to Noise}

Experiments introducing noise to the evaluation schemes showed that late fusion with DST was the most robust scheme. This finding suggests that when noise is present in sensor measurements 
(e.g., due to Bluetooth signal remixing, wireless signal interferences, and potential hardware failure), the late fusion with DST scheme should be used. Generally, the late fusion schemes (either average or DST voting) were more robust to noise than early fusion schemes. This observation echoed a commonly acknowledged advantage of late fusion in its robustness to noise [94]. Interestingly, the early fusion with ICA approach exhibited the worst performance in noise robustness, even though it achieved the optimal performance without noise. Early fusion techniques and ICA algorithms resulted in features based on the interaction of different raw features. The addition of noise may have been amplified in the complicated process and significantly degraded the final performance. Therefore, the selection of the optimal fusion strategy needs to consider the environmental conditions and constraints. However, noise assessment in this study was simulated, and further research is needed to assess findings with "real-life" noise distributions.

\subsection{Model Generalizations}

This article focused on the RAS scenario to develop and evaluate the workload recognition algorithm. Similar research has been conducted in other fields, such as HCI, driving, and flight control. The performance trends of individual sensors in this study were aligned with the findings from other areas, such as driving [87, 88], flight control [90], first-person shooting simulation [95], and so on. When setting up the workload prediction algorithm, the specific domain information was not used explicitly in training, therefore, this approach may be generalizable to other domains. In addition, the trained workload prediction algorithm may also be generalizable using certain techniques such as transfer learning with domain adaptation to fine-tune the developed algorithm to the new domain.

Different domains can have varying effect on sensor measurement reliability. In RAS, the surgeon is required to sit on the console and remain still most of the time. Therefore, high-quality EEG, EMG, and EDA signals can be acquired. In other domains with large body movements, EEG, EMG, and EDA signal quality may be compromised due to the noise introduced due to body movements. For example, this would be the case when using touchless interfaces to manipulate magnetic resonance images in the OR [96]. In those scenarios, the HRV and MOV signals may be more reliable and desired for workload prediction. Specifically, these signals may be less susceptible to motion (they are designed for dynamic activities) and may be able to capture the large motions that better differentiate workload levels in more dynamic tasks.

Sample size was a limitation of the current work; however, this pilot study provides an initial investigation for the different configurations of the workload prediction algorithm (e.g., classification algorithms, fusion scheme, and modality comparisons) in the RAS domain. Another limitation was the assumption of constant workload throughout the execution of the simulation tasks. Further work is needed to develop novel tasks that sustain workload during tasks relevant to the RAS domain.

\subsection{Integration into the Entire Robotic System}

This article focused discussion on the design and development of the workload prediction algorithm. The algorithm, once deployed, can be used in the RAS system to increase surgical performance. Even though the integration of the developed algorithm into the complete robotic system requires more work and goes beyond the scope of this current study, we would still like to discuss potential integration methods as well as required future studies.

We proposed two approaches for integrating the workload prediction algorithm into the entire robotic system: one is from the training perspective and the other is from the real-time operation perspective. From the training perspective, the workload levels can be recorded and evaluated as part of the training curriculum to help the trainee identify the most stressful tasks/skills to guide 
individualized practice. This has the potential to improve both practice effectiveness and speed. Workload level can also be integrated into an evaluation system to evaluate the trainee's learning outcomes, and previous work found that cognitive workload levels were associated with expertise levels. From the second point of view on real-time operations, the workload prediction system can integrate into the robot actions directly during field surgeries. Once the workload prediction system has identified a long period of intense workload levels from the operating surgeon, the system can perform several actions such as remind the surgeon to pause and ask for extra assistance, pause the RAS system to avoid potential damage to the patient, or trigger shared autonomy mode to provide extra low-level assistance such as tremor removal. Depending on the actual use case, the requirement on the classification accuracy would be different. The proposed method can achieve an accuracy of $83.2 \%$. If the developed algorithm is going to be used during surgery in a shared autonomy mode, then the accuracy level might not be enough and additional work is needed to improve accuracy. However, if the developed algorithm is going to be used for training pre-med students, then lower accuracy may be sufficient. Moreover, several sensing modalities did not significantly increase workload prediction and can be removed to improve usability. To summarize, additional field studies are needed to integrate the workload prediction algorithm into the entire system.

\section{CONCLUSIONS}

Human workload measurement is necessary for designing adaptive user interfaces. In the teleoperation domain, intraoperative cognitive load assessment is necessary for reliable human-machine collaboration. Current questionnaire-type solutions (e.g., NASA-TLX) are subjective and cannot measure cognitive workload non-intrusively. To tackle this challenge, our article proposed a computational framework that leverages multimodal human sensing and machine learning algorithms to predict human cognitive workload. The proposed framework was evaluated in telerobotics with the da Vinci Skills Simulator, where twelve surgeons participated and performed surgical training tasks of different workload levels. Our algorithm accurately predicted surgeons' workload $83.2 \%$ of the time, using the optimal classifier, modality, and sensor fusion scheme. Specifically, early multimodal fusion with ICA analysis outperformed all fusion schemes in an accurate sensor reading condition. When artificial noise was introduced into the sensor readings, the late multimodal fusion with DST was found to be most resilient and robust to the disturbance. Therefore, it is important to select the most appropriate fusion scheme for best performance based on the noise level and sensor reliability. The successful design and implementation of the cognitive workload assessment framework provide the foundation for dynamic interface design.

\section{APPENDIX}

Table A1. Absolute F1 Score of Different Fusion Schemes with Different Noise Levels

\begin{tabular}{|c|c|c|c|c|c|c|c|}
\hline Fusion $\backslash \boldsymbol{\sigma}$ & no noise & $\mathbf{0 . 1}$ & $\mathbf{0 . 2}$ & $\mathbf{0 . 3}$ & $\mathbf{0 . 4}$ & $\mathbf{0 . 5}$ & Average \\
\hline Early & 0.716 & 0.660 & 0.646 & 0.653 & 0.639 & 0.66 & 0.652 \\
\hline Early $\left(\mathrm{ICA}_{10}\right)$ & 0.811 & 0.635 & 0.561 & 0.55 & 0.549 & 0.536 & 0.566 \\
\hline Late $(\mathrm{avg})$ & 0.714 & 0.674 & 0.681 & 0.695 & 0.708 & 0.722 & 0.696 \\
\hline Late $(\mathrm{DST})$ & 0.740 & 0.714 & 0.735 & 0.735 & 0.747 & 0.760 & $\mathbf{0 . 7 3 8}$ \\
\hline
\end{tabular}


Table A2. PRR of Different Fusion Schemes with Different Noise Levels

\begin{tabular}{|c|c|c|c|c|c|c|c|}
\hline Fusion $\backslash \boldsymbol{\sigma}$ & no noise & $\mathbf{0 . 1}$ & $\mathbf{0 . 2}$ & $\mathbf{0 . 3}$ & $\mathbf{0 . 4}$ & $\mathbf{0 . 5}$ & Average \\
\hline Early & $100 \%$ & $92.18 \%$ & $90.22 \%$ & $91.20 \%$ & $89.25 \%$ & $92.18 \%$ & $91.01 \%$ \\
\hline Early $\left(\mathrm{ICA}_{10}\right)$ & $100 \%$ & $78.30 \%$ & $69.17 \%$ & $67.82 \%$ & $67.69 \%$ & $66.09 \%$ & $69.82 \%$ \\
\hline Late (avg) & $100 \%$ & $94.40 \%$ & $95.38 \%$ & $97.34 \%$ & $99.16 \%$ & $89.36 \%$ & $95.13 \%$ \\
\hline Late (DST) & $100 \%$ & $96.49 \%$ & $99.32 \%$ & $99.32 \%$ & $100.95 \%$ & $102.70 \%$ & $\mathbf{9 9 . 7 6 \%}$ \\
\hline
\end{tabular}

\section{REFERENCES}

[1] L. M. Hirshfield et al. 2009. Brain measurement for usability testing and adaptive interfaces: An example of uncovering syntactic workload with functional near infrared spectroscopy. In Proceedings of the SIGCHI Conference on Human Factors in Computing Systems 2009, 2185-2194.

[2] J. Ren, R. V. Patel, K. A. McIsaac, G. Guiraudon, and T. M. Peters. 2008. Dynamic 3-D virtual fixtures for minimally invasive beating heart procedures. IEEE Trans. Med. Imaging 27, 8 (2008), 1061-1070.

[3] R. Taylor et al. 1999. A steady-hand robotic system for microsurgical augmentation. Int. f. Robot. Res. 18, 12 (1999), 1201-1210.

[4] Y. Gao et al. 2014. JHU-ISI gesture and skill assessment working set (JIGSAWS): A surgical activity dataset for human motion modeling. In Proceedings of the International Conference on Medical Image Computing and Computer Assisted Intervention (M2CAI'14). 3.

[5] T. Akinbiyi et al. 2006. Dynamic augmented reality for sensory substitution in robot-assisted surgical systems. In Proceedings of the 28th Annual International Conference of the IEEE on Engineering in Medicine and Biology Society (EMBS'06). 567-570.

[6] R. C. Britt, M. W. Scerbo, M. Montano, R. A. Kennedy, E. Prytz, and D. Stefanidis. 2015. Intracorporeal suturing: Transfer from fundamentals of laparoscopic surgery to cadavers results in substantial increase in mental workload. Surgery 158, 5 (2015), 1428-1433.

[7] P. Crochet, A. Agostini, S. Knight, N. Resseguier, S. Berdah, and R. Aggarwal. 2017. The performance gap for residents in transfer of intracorporeal suturing skills from box trainer to operating room. F. Surg. Educ. 74, 6 (2017), 1019-1027.

[8] A. A. Hussein et al. 2016. Technical mentorship during robot-assisted surgery: A cognitive analysis. BfU Int. 118, 3 (2016), 429-436.

[9] S. G. Hart and L. E. Staveland. 1988. Development of NASA-TLX (task load index): Results of empirical and theoretical research. Adv. Psychol. 52, (1988), 139-183.

[10] G. B. Reid and T. E. Nygren. 1988. The subjective workload assessment technique: A scaling procedure for measuring mental workload. Adv. Psychol. 52, 185-218.

[11] S. Rubio, E. Díaz, J. Martín, and J. M. Puente. 2004. Evaluation of subjective mental workload: A comparison of SWAT, NASA-TLX, and workload profile methods. Appl. Psychol. 53, 1 (2004), 61-86.

[12] M. W. Scerbo, R. A. Kennedy, M. Montano, R. C. Britt, S. S. Davis, and D. Stefanidis. 2013. A spatial secondary task for measuring laparoscopic mental workload: Differences in surgical experience. In Proceedings of the Human Factors and Ergonomics Society Annual Meeting, Vol. 57, 728-732.

[13] R. C. Grant, C. M. Carswell, C. H. Lio, and W. B. Seales. 2013. Measuring surgeons' mental workload with a time-based secondary task. Ergon. Des. 21, 1 (2013), 7-11.

[14] A. Darzi, S. Smith, and N. Taffinder. 1999. Assessing operative skill: Needs to become more objective. BMJ 318, 7188, 887, 1999.

[15] P. Nickel and F. Nachreiner. 2003. Sensitivity and diagnosticity of the 0.1 -hz component of heart rate variability as an indicator of mental workload. Hum. Factors 45, 4 (2003), 575-590.

[16] G. Durantin, J.-F. Gagnon, S. Tremblay, and F. Dehais. 2014. Using near infrared spectroscopy and heart rate variability to detect mental overload. Behav. Brain Res. 259, Supplement C, 16-23.

[17] P. G. Jorna. Spectral analysis of heart rate and psychological state: A review of its validity as a workload index. Biol. Psychol. 34, 2 (1992), 237-257.

[18] N. Meshkati. 1988. Heart rate variability and mental workload assessment. In Advances in Psychology, Vol. 52, Elsevier, 101-115.

[19] G. F. Wilson. 2002. An analysis of mental workload in pilots during flight using multiple psychophysiological measures. Int. F. Aviat. Psychol. 12, 1 (2002), 3-18.

[20] P. Ghaderyan and A. Abbasi. 2016. An efficient automatic workload estimation method based on electrodermal activity using pattern classifier combinations. Int. F. Psychophysiol. 110, Supplement C, 91-101. 
[21] B. Mehler, B. Reimer, J. Coughlin, and J. Dusek. 2009. Impact of incremental increases in cognitive workload on physiological arousal and performance in young adult drivers. Transp. Res. Rec. f. Transp. Res. Board, 2138, 6-12.

[22] C. Berka et al. 2007. EEG correlates of task engagement and mental workload in vigilance, learning, and memory tasks. Aviat. Space Environ. Med. 78, 5 (2007), B231-B244.

[23] T. C. Hankins and G. F. Wilson. 1998. A comparison of heart rate, eye activity, EEG and subjective measures of pilot mental workload during flight. Aviat. Space Environ. Med. 69, 4 (1998), 360-367.

[24] F. J. Pérez-Duarte, M. Lucas-Hernández, A. Matos-Azevedo, J. A. Sánchez-Margallo, I. Díaz-Güemes, and F. M. Sánchez-Margallo. 2014. Objective analysis of surgeons' ergonomy during laparoendoscopic single-site surgery through the use of surface electromyography and a motion capture data glove. Surg. Endosc. 28, 4 (2014), 1314-1320.

[25] G. Lee, E. Sutton, T. Clanton, and A. Park. 2011. Higher physical workload risks with NOTES versus laparoscopy: A quantitative ergonomic assessment. Surg. Endosc. 25, 5 (2011), 1585-1593.

[26] S. G. Hart and L. E. Staveland. 1988. Development of NASA-TLX (Task Load Index): Results of empirical and theoretical research. Adv. Psychol. 52, 139-183.

[27] M. R. Wilson, J. M. Poolton, N. Malhotra, K. Ngo, E. Bright, and R. S. Masters. 2011. Development and validation of a surgical workload measure: The surgery task load index (SURG-TLX). World f. Surg. 35, 9 (2011).

[28] D. Yu et al. 2017. Intraoperative workload in robotic surgery assessed by wearable motion tracking sensors and questionnaires. Surg. Endosc 31, 2 (2017), 877-886.

[29] P. S. Tsang and V. L. Velazquez. 1996. Diagnosticity and multidimensional subjective workload ratings. Ergonomics 39, 3 (1996), 358-381.

[30] J. A. Martin et al. 1997. Objective structured assessment of technical skill (OSATS) for surgical residents. Br. F. Surg. 84, 2 (1997), 273-278.

[31] E. T. Solovey, M. Zec, E. A. Garcia Perez, B. Reimer, and B. Mehler. 2014. Classifying driver workload using physiological and driving performance data: Two field studies. In Proceedings of the 32nd Annual ACM Conference on Human Factors in Computing Systems. 4057-4066.

[32] D. Novak, B. Beyeler, X. Omlin, and R. Riener. 2014. Workload estimation in physical human-robot interaction using physiological measurements. Interact. Comput. 27, 6 (2014), 616-629, 2014.

[33] K. A. Guru, S. B. Shafiei, A. Khan, A. A. Hussein, M. Sharif, and E. T. Esfahani. 2015. Understanding cognitive performance during robot-assisted surgery. Urology 86, 4 (2015), 751-757.

[34] H. Aghajani, M. Garbey, and A. Omurtag. 2017. Measuring mental workload with EEG+ fNIRS. Front. Hum. Neurosci. 11359.

[35] F. A. Muckler and S. A. Seven. 1992. Selecting performance measures: Objective versus subjective measurement, Hum. Factors 34, 4 (1992), 441-455.

[36] D. A. Wiegmann, A. W. ElBardissi, J. A. Dearani, R. C. Daly, and T. M. Sundt. 2007. Disruptions in surgical flow and their relationship to surgical errors: An exploratory investigation. Surgery 142, 5 (2007), 658-665.

[37] B. Zheng, D. V. Martinec, M. A. Cassera, and L. L. Swanström. 2008. A quantitative study of disruption in the operating room during laparoscopic antireflux surgery. Surg. Endosc. 22, 10 (2008), 2171-2177.

[38] N. Sevdalis, D. Forrest, S. Undre, A. Darzi, and C. Vincent. 2008. Annoyances, disruptions, and interruptions in surgery: the disruptions in surgery index (DiSI). World f. Surg. 32, 8 (2008), 1643-1650.

[39] M. C. Vassiliou et al. 2005. A global assessment tool for evaluation of intraoperative laparoscopic skills. Am. f. Surg 190, 1 (2005), 107-113.

[40] M. R. Polin et al. 2016. Crowdsourcing: A valid alternative to expert evaluation of robotic surgery skills. Am. F. Obstet. Gynecol. 215, 5 (2016), 644-e1.

[41] J. B. Brookings, G. F. Wilson, and C. R. Swain. 1996. Psychophysiological responses to changes in workload during simulated air traffic control. Biol. Psychol 42, 3 (1996), 361-377.

[42] G. Borghini, L. Astolfi, G. Vecchiato, D. Mattia, and F. Babiloni. 2014. Measuring neurophysiological signals in aircraft pilots and car drivers for the assessment of mental workload, fatigue and drowsiness. Neurosci. Biobehav. Rev. 44, Supplement C, 58-75.

[43] K. A. Guru et al. 2015. Cognitive skills assessment during robot-assisted surgery: Separating the wheat from the chaff. BfU Int. 115, 1 (2015), 166-174.

[44] L. W. Freedman, A. S. Scerbo, M. E. Dawson, A. Raine, W. O. McCLURE, and P. H. Venables. 1994. The relationship of sweat gland count to electrodermal activity. Psychophysiology 31, 2 (1994), 196-200.

[45] Y. Shimomura, T. Yoda, K. Sugiura, A. Horiguchi, K. Iwanaga, and T. Katsuura. 2008. Use of frequency domain analysis of skin conductance for evaluation of mental workload. F. Physiol. Anthropol. 27, 4 (2008), 173-177.

[46] B. Reimer, B. Mehler, J. F. Coughlin, K. M. Godfrey, and C. Tan. 2009. An on-road assessment of the impact of cognitive workload on physiological arousal in young adult drivers. In Proceedings of the 1st International Conference on Automotive User Interfaces and Interactive Vehicular Applications. 115-118. 
[47] D. W. Rowe, J. Sibert, and D. Irwin. 1998. Heart rate variability: Indicator of user state as an aid to human-computer interaction. In Proceedings of the SIGCHI Conference on Human Factors in Computing Systems. 480-487.

[48] D. Chen and R. Vertegaal. Using mental load for managing interruptions in physiologically attentive user interfaces. In Proceedings of the CHI'04: Extended Abstracts on Human Factors in Computing Systems. 1513-1516.

[49] A. J. Tattersall and G. R. J. Hockey. 1995. Level of operator control and changes in heart rate variability during simulated flight maintenance. Hum. Factors 37, 4 (1995), 682-698.

[50] A. H. Roscoe. 1992. Assessing pilot workload. Why measure heart rate, HRV and respiration? Biol. Psychol 34, 2 (1992), 259-287.

[51] D. De Waard. 1996. The Measurement of Drivers' Mental Workload. Groningen University, Traffic Research Center Netherlands.

[52] R. Berguer, W. D. Smith, and Y. H. Chung. 2001. Performing laparoscopic surgery is significantly more stressful for the surgeon than open surgery. Surgical Endoscopy, 15, 10 (2001), 1204-1207. Google Scholar. Retrieved from https://scholar.google.com/scholar?hl=en\&q=Berguer $\% 2 \mathrm{C}+\mathrm{R} . \% 2 \mathrm{C}+$ Smith $\% 2 \mathrm{C}+\mathrm{W} .+\mathrm{D} . \% 2 \mathrm{C}+\% 26+\mathrm{Chung} \% 2 \mathrm{C}+\mathrm{Y} .+\mathrm{H}$. $+\% 282001 \% 29$.+Performing+laparoscopic + surgery+is+significantly+more+stressful+for+the+surgeon+than + open + surgery.+Surgical+endoscopy $\% 2 \mathrm{C}+15 \% 2810 \% 29 \% 2 \mathrm{C}+1204-1207 . \& \mathrm{btnG}=\&$ as_sdt $=1 \% 2 \mathrm{C} 15 \&$ as_sdtp $=$.

[53] P. Zhang, X. Wang, J. Chen, and W. You. 2017. Feature weight driven interactive mutual information modeling for heterogeneous bio-signal fusion to estimate mental workload. Sensors 17, 10 (2017), 2315.

[54] G. F. Wilson and C. A. Russell. 2003. Real-time assessment of mental workload using psychophysiological measures and artificial neural networks. Hum. Factors 45, 4 (2003), 635-644.

[55] Y. Liu, H. Ayaz, and P. A. Shewokis. 2017. Multisubject 'learning' for mental workload classification using concurrent EEG, fnirs, and physiological measures. Front. Hum. Neurosci. 11, 389

[56] A. Jimenez-Molina, C. Retamal, and H. Lira. 2018. Using psychophysiological sensors to assess mental workload during web browsing. Sensors 18, 2 (2018), 458.

[57] "Mimic Simulation | Leader in Robotic Surgery Simulation \& Training," Mimic Simulation. Retrieved from http:// mimicsimulation.com/.

[58] M. Spüler, C. Walter, W. Rosenstiel, P. Gerjets, K. Moeller, and E. Klein. 2016. EEG-based prediction of cognitive workload induced by arithmetic: A step towards online adaptation in numerical learning. ZDM, 48, 3 (2016), 267-278.

[59] J. Fan et al. 2015. A step towards EEG-based brain computer interface for autism intervention. In Proceedings of the 37th Annual International Conference of the IEEE on Engineering in Medicine and Biology Society (EMBC'15). 3767-3770.

[60] M. Teplan. 2002. Fundamentals of EEG measurement. Meas. Sci. Rev. 2, 2 (2002), 1-11.

[61] S. F. Liang, C. T. Lin, R. C. Wu, Y. C. Chen, T. Y. Huang, and T. P. Jung. 2006. Monitoring driver's alertness based on the driving performance estimation and the EEG power spectrum analysis. In Proceedings of the 27th Annual International Conference of the Engineering in Medicine and Biology Society (IEEE-EMBS'05). 5738-5741.

[62] EEG alpha and theta oscillations reflect cognitive and memory performance: A review and analysis-ScienceDirect. Retrieved from http://www.sciencedirect.com/science/article/pii/S0165017398000563.

[63] A. Delorme and S. Makeig. 2004. EEGLAB: An open source toolbox for analysis of single-trial EEG dynamics including independent component analysis. 7. Neurosci. Methods 134, 1 (2004), 9-21.

[64] M. D’Alessandro, R. Esteller, G. Vachtsevanos, A. Hinson, J. Echauz, and B. Litt. 2003. Epileptic seizure prediction using hybrid feature selection over multiple intracranial EEG electrode contacts: a report of four patients. IEEE Trans. Biomed. Eng. 50, 5 (2003), 603-615.

[65] S. Güneş, K. Polat, and Ş. Yosunkaya. 2010. Efficient sleep stage recognition system based on EEG signal using k-means clustering-based feature weighting. Expert Syst. Appl. 37, 12 (2010), 7922-7928.

[66] M. Benedek and C. Kaernbach. 2010. Decomposition of skin conductance data by means of nonnegative deconvolution. Psychophysiology 47, 4 (2010), 647-658.

[67] D. R. Bach. 2014. A head-to-head comparison of SCRalyze and ledalab, two model-based methods for skin conductance analysis. Biol. Psychol. 103, 63-68.

[68] W. Boucsein. 2012. Electrodermal Activity. Springer Science \& Business Media.

[69] A. J. Casson, A. Vazquez Galvez, and D. Jarchi. 2016. Gyroscope vs. accelerometer measurements of motion from wrist PPG during physical exercise. ICT Express 2, 4 (2016), 175-179.

[70] A. Rieger, R. Stoll, S. Kreuzfeld, K. Behrens, and M. Weippert. 2014. Heart rate and heart rate variability as indirect markers of surgeons' intraoperative stress. Int. Arch. Occup. Environ. Health 87, 2 (2014), 165-174.

[71] B. Mehler, B. Reimer, and Y. Wang. 2011. A comparison of heart rate and heart rate variability indices in distinguishing single-task driving and driving under secondary cognitive workload. In Proceedings of the 6th International Driving Symposium on Human Factors in Driver Assessment, Training and Vehicle Design. 590-597.

[72] D. A. Belsley, E. Kuh, and R. E. Welsch. 2005. Regression Diagnostics: Identifying Influential Data and Sources of Collinearity. John Wiley \& Sons, 571. 
[73] G. Srivastava, S. Crottaz-Herbette, K. M. Lau, G. H. Glover, and V. Menon. 2005. ICA-based procedures for removing ballistocardiogram artifacts from EEG data acquired in the MRI scanner. Neuroimage 24, 1 (2005), 50-60.

[74] H. P. Martínez and G. N. Yannakakis. 2014. Deep multimodal fusion: Combining discrete events and continuous signals. In Proceedings of the 16th International Conference on Multimodal Interaction 2014, 34-41.

[75] T. Zhou and J. P. Wachs. 2017. Early prediction for physical human robot collaboration in the operating room. Auton. Robots 1-19.

[76] G. Shafer. 2016. Dempster's rule of combination. Int. F. Approx. Reason. 79, 26-40.

[77] J. Zhang, Z. Yin, and R. Wang. 2015. Recognition of mental workload levels under complex human-machine collaboration by using physiological features and adaptive support vector machines. IEEE Trans. Hum.-Mach. Syst. 45, 2 (2015), 200-214.

[78] S. G. Hart. 2006. NASA-task load index (NASA-TLX); 20 years later. In Proceedings of the Human Factors and Ergonomics Society Annual Meeting, Vol. 50, 904-908.

[79] K. T. Finnegan, A. M. Meraney, I. Staff, and S. J. Shichman. 2012. da Vinci skills simulator construct validation study: Correlation of prior robotic experience with overall score and time score simulator performance. Urology 80, 2 (2012), 330-336.

[80] F. T. Eggemeier and G. F. Wilson. 1991. Performance-based and subjective assessment of workload in multi-task environments. Mult.-Task Perform. 217-278.

[81] da Vinci Surgery-Minimally Invasive Robotic Surgery with the da Vinci surgical system. Retrieved from http://www. davincisurgery.com/.

[82] D. Gopher and R. Braune. 1984. On the psychophysics of workload: Why bother with subjective measures? Hum. Factors 26, 5 (1984), 519-532.

[83] J. R. Landis and G. G. Koch. 1977. The measurement of observer agreement for categorical data. Biometrics 159-174, 1977.

[84] Y. Saeys, I. Inza, and P. Larrañaga. 2007. A review of feature selection techniques in bioinformatics. Bioinformatics 23, 19 (2007), 2507-2517.

[85] D. M. Powers. 2011. Evaluation: From precision, recall, and F-measure to ROC, informedness, markedness, and correlation. Journal of Machine Learning Technologies 2, 1 (2011), 37-63. https://dspace2.flinders.edu.au/xmlui/handle/ $2328 / 27165$.

[86] J. Bergstra and Y. Bengio. 2012. Random search for hyper-parameter optimization. Ұ. Mach. Learn. Res. 13, 1 (2012), 281-305.

[87] F. Putze, J.-P. Jarvis, and T. Schultz. 2010. Multimodal recognition of cognitive workload for multitasking in the car. In Proceedings of the 20th International Conference on Pattern Recognition (ICPR'10). 3748-3751.

[88] J. Jarvis, F. Putze, D. Heger, and T. Schultz. 2011. Multimodal person independent recognition of workload related biosignal patterns. In Proceedings of the 13th International Conference on Multimodal Interfaces. 205-208.

[89] M. A. Hogervorst, A.-M. Brouwer, and J. B. van Erp. 2014. Combining and comparing EEG, peripheral physiology and eye-related measures for the assessment of mental workload. Front. Neurosci. 8, 322, 2014.

[90] P. Aricò et al. 2014. Towards a multimodal bioelectrical framework for the online mental workload evaluation. In Proceedings of the 36th Annual International Conference of the IEEE on Engineering in Medicine and Biology Society (EMBC'14). 3001-3004.

[91] S. McCrea, G. Geršak, and D. Novak. 2016. Absolute and relative user perception of classification accuracy in an affective video game. Interact. Comput. 29, 2 (2016), 271-286.

[92] A. T. Kamzanova, A. M. Kustubayeva, and G. Matthews. 2014. Use of EEG workload indices for diagnostic monitoring of vigilance decrement. Hum. Factors 56, 6 (2014), 1136-1149.

[93] C. Dussault, J.-C. Jouanin, M. Philippe, and C.-Y. Guezennec. 2005. EEG and ECG changes during simulator operation reflect mental workload and vigilance. Aviat. Space Environ. Med. 76, 4 (2005), 344-351.

[94] S. Rossi, E. Leone, M. Fiore, A. Finzi, and F. Cutugno. 2013. An extensible architecture for robust multimodal humanrobot communication. In Proceedings of the IEEE/RSf International Conference on Intelligent Robots and Systems (IROS'13). 2208-2213.

[95] L. J. Trejo, N. J. McDonald, R. Matthews, and B. Z. Allison. 2007. Experimental design and testing of a multimodal cognitive overload classifier. Found. Augment. Cogn. 13-22.

[96] M. Jacob, C. Cange, R. Packer, and J. P. Wachs. 2012. Intention, context and gesture recognition for sterile MRI navigation in the operating room. In Progress in Pattern Recognition, Image Analysis, Computer Vision, and Applications, L. Alvarez, M. Mejail, L. Gomez, and J. Jacobo, Eds. Springer, Berlin, 220-227.

Received April 2018; revised July 2019; accepted October 2019 